\title{
STABLE PENCILS OF HYPERBOLIC POLYNOMIALS AND THE CAUCHY PROBLEM FOR HYPERBOLIC EQUATIONS WITH A SMALL PARAMETER AT THE HIGHEST DERIVATIVES
}

\author{
L. R. VOLEVICH AND E. V. RADKEVICH
}

\begin{abstract}
We study pencils of hyperbolic polynomials of the form $\mathcal{R}(\tau, \xi)=$ $\sum_{j=0}^{N}(-i)^{j} \gamma_{j} P_{j}(\tau, \xi)$, where $P_{j}(\tau, \xi)$ is a real homogeneous polynomials of degree $m-j$ resolved with respect to the highest power of $\tau$ and $P_{j}(1,0)=1$; the numbers $\gamma_{0}, \ldots, \gamma_{N}$ are positive. In the first part of the paper we find necessary and close to sufficient conditions of stability of the polynomial $\mathcal{R}(\tau, \xi)$ (i.e., the condition that its roots $\tau_{j}(\xi)$ lie in the open upper half-plane of the complex plane). This problem is closely related to the problem on uniform (with respect to a small parameter) estimates for the solution of the Cauchy problem for hyperbolic equations with a small parameter. The latter problem (both for constant and variable coefficients) is the topic of the second part of the paper.
\end{abstract}

\section{CONTEnts}

1. Introduction

2. The Hermite-Biehler Theorem and hyperbolic pairs

3. Notes about the roots of the polynomial $\mathcal{P}$ and $\mathcal{Q}$

4. Stable hyperbolic pencils

5. Stability conditions for triples and quadruples of hyperbolic polynomials

6. Strictly hyperbolic polynomials with a small and a large parameter

7. The Cauchy problem for a hyperbolic equations with a small parameter at highest derivatives

8. The proof of energy estimates

9. Pencils related to the system of moments for the kinetic Fokker-Planck equation

\section{INTRODUCTION}

The subject matter of this article was suggested by the problem of asymptotic stability of the Cauchy problem for certain classes of partial differential equations with constant coefficients, in particular, for the Grad lattices 1, 2], which are used as approximations for the kinetic Boltzmann and Fokker-Planck equations.

We consider the system

$$
L\left(\frac{\partial}{\partial t}, \frac{\partial}{\partial x}, \lambda\right) U(t, x):=I \frac{\partial U(t, x)}{\partial t}+\sum_{j=1}^{n} A^{(j)} \frac{\partial U(t, x)}{\partial x_{j}}+\lambda A^{(0)} U(t, x)=0
$$

Here $x=\left(x_{1}, \ldots, x_{n}\right) \in \mathbb{R}^{n}$ and $\frac{\partial}{\partial x}=\left(\frac{\partial}{\partial x_{1}}, \ldots, \frac{\partial}{\partial x_{n}}\right), A^{(0)}, A^{(1)}, \ldots, A^{(n)}$ are constant real $m \times m$ matrices, $I$ is the identity $m \times m$ matrix, and $\lambda$ is a large positive parameter.

2000 Mathematics Subject Classification. Primary 35B25, 35L25.

The first author was supported by the Russian Foundation for Basic Research (Grant 03-01-00189) and INTAS (Project no. 899). The second author was supported by the Russian Foundation for Basic Research (Grant 03-01-00189). 
If a vector-valued function $U(t, x)$ is a solution of the system (1.1), then each component $u(t, x)$ of this function is a solution of the scalar equation

$$
R\left(\frac{\partial}{\partial t}, \frac{\partial}{\partial x}, \lambda\right) u(t, x):=\operatorname{det} L\left(\frac{\partial}{\partial t}, \frac{\partial}{\partial x}, \lambda\right) u(t, x)=0,
$$

and the analysis of asymptotic stability of the Cauchy problem for the system (1.1) is reduced to the similar question for the scalar operator (1.2).

It is well known that each solution of the scalar equation (1.2) can be represented as a Fourier integral

$$
(2 \pi)^{-n / 2} \int_{\mathbb{R}^{n}} h(\xi, \lambda) \exp \{i(\xi x+\tau(\xi, \lambda) t)\} d \xi,
$$

where $\tau=\tau(\xi, \lambda)$ is a root of the dispersion equation

$$
\mathcal{R}(\tau, \xi, \lambda):=i^{-m} R(i \tau, i \xi, \lambda)=R(\tau, \xi,-i \lambda)=0 .
$$

A pencil of polynomials of the form (1.3) is called totally stable if for each $\xi \in \mathbb{R}^{n}$ all roots (1.3) lie in the open upper half-plane of the complex plane.

The total stability condition guarantees the asymptotic stability of the Cauchy problem for the initial system in any reasonable norm. However, the total stability condition does not hold in many interesting examples of the system (1.1). Indeed, total stability means that equation (1.3) has no roots in the closed lower half-plane. This implies that the matrix $A^{(0)}$ in (1.1) is nonsingular. This condition does not look natural. For example, in the case of the Grad lattice for the Fokker-Planck equation, the matrix $A^{(0)}$ is diagonal and one of its diagonal entries equals 0 (see $\S 9$ ).

Because of this, we will use a weaker notion of stability.

Definition 1.1. A pencil of polynomials (1.3) is called stable if

$$
\mathcal{R}(\tau, \xi, \lambda) \neq 0, \quad \operatorname{Im} \tau \leq 0, \quad|\tau|+|\xi|>0 .
$$

This condition is sufficient for the asymptotic stability of the Cauchy problem for the system (1.1) in reasonable integral norms, e.g., in the $L^{2}$-norm.

New let us analyze in more detail the form of the polynomial in the dispersion equation (1.3). We rewrite it as a polynomial in $-i \lambda$ with coefficients depending on the variables $(\tau, \xi)$ :

$$
\mathcal{R}(\tau, \xi, \lambda)=\sum_{j=0}^{m}(-i \lambda)^{j} P_{j}(\tau, \xi) .
$$

Let us discuss properties of the polynomials $P_{j}(\tau, \xi), j=0, \ldots, N$ in (1.5).

1. The polynomials $P_{j}(\tau, \xi), j=0, \ldots, m$, are real and homogeneous, with the homogeneity degree of $P_{j}$ being $m-j$.

The form of the matrix operator (1.1) shows that the polynomial $P_{0}(\tau, \xi)$ is resolved with respect to the highest power of $\tau$, with the corresponding coefficient equal to 1, i.e.,

$$
P_{0}(\tau, \xi)=\tau^{m}+\sum_{|\alpha|=k \geq 1} p_{k \alpha} \tau^{m-k} \xi^{\alpha} .
$$

Now we consider the sequence of coefficients $\left\{P_{j}(1,0)\right\}$, where $j=0,1, \ldots, m$. Denote by $N$ the largest index for which $P_{N}(1,0) \neq 0$, and assume that $N<m$. The case $N=m$ is much simpler and we will not dwell on it. We note also that in all our examples the first condition holds.

For the system (1.1), we assume additionally that

$$
P_{N+1}(\tau, \xi) \equiv 0, \quad \ldots, \quad P_{m}(\tau, \xi) \equiv 0 .
$$


Under this assumption, the polynomial in (1.3) has the form

$$
\mathcal{R}(\tau, \xi, \lambda)=\sum_{j=0}^{N}(-i \lambda)^{j} P_{j}(\tau, \xi),
$$

with the last polynomial in the sum (1.7) resolved with respect to the highest power of $\tau$ :

$$
P_{N}(\tau, \xi)=\gamma_{N} \tau^{m-N}+\sum_{|\alpha|=k \geq 1} q_{k \alpha} \tau^{m-N-k} \xi^{\alpha}, \quad \gamma_{N} \neq 0 .
$$

Taking $\xi=0$ in (1.7) and dividing by $\tau^{m-N}$, we obtain a polynomial in $\tau$ :

$$
r(\tau, \lambda):=\tau^{N}+\sum_{j=1}^{N}(-i \lambda)^{j} \gamma_{j} \tau^{N-j}, \quad \gamma_{j}=P_{j}(1,0)
$$

Definition 1.2. The polynomial $r(\tau)$ depending on $\tau \in \mathbb{C}$ is said to be stable if all its roots lie in the open upper half-plane of the complex plane.

The definition immediately implies the following result.

Lemma 1.1. Let conditions (1.6) and (1.8) hold. Then the polynomial (1.7) satisfies condition (1.4),

$$
\mathcal{R}(\tau, \xi, \lambda) \neq 0, \quad \operatorname{Im} \tau \leq 0 \quad \forall \xi \in \mathbb{R}^{n} \backslash\{0\},
$$

and the polynomial (1.9) is stable,

$$
r(\tau, \lambda) \neq 0, \quad \operatorname{Im} \tau \leq 0 .
$$

We note that according to [3] the stability of the polynomial $r(\tau)$ implies that

$$
\lambda^{j} \gamma_{j}>0, \quad j=1, \ldots, N
$$

Since the parameter $\lambda$ is positive, we have

$$
\gamma_{j}>0, \quad j=1, \ldots, N .
$$

Therefore the stability condition (1.4) results in an important restriction on the system under consideration:

2. All polynomials $P_{j}, j=0, \ldots, N$, are resolved with respect to the highest power of $\tau$.

Remark 1.1. Conditions (1.10) are only necessary for the stability of the polynomials $r(\tau, \lambda)$. Necessary and sufficient conditions for the coefficients $\gamma_{j}$ are given by the RouthHurwitz criterion (see [3]) and these conditions do not depend on $\lambda$. For $N=1,2$ this criterion does not contain conditions different from (1.10). For $N=3$ the Routh-Hurwitz criterion contains an additional condition

$$
\gamma_{3}<\gamma_{1} \gamma_{2} .
$$

For $N=4$ there are two additional conditions:

$$
\gamma_{3}<\gamma_{1} \gamma_{2}, \quad \gamma_{4}<\frac{\gamma_{3}}{\gamma_{1}^{2}}\left(\gamma_{1} \gamma_{2}-\gamma_{3}\right) .
$$

The above arguments suggest that it is natural to use slightly modified notations, replacing the polynomials $P_{j}(\tau, \xi)$ with $\gamma_{j} P_{j}(\tau, \xi)$, where the polynomials $P_{j}(\tau, \xi)$ are resolved with respect to the highest power of $\tau$ and the corresponding coefficients are equal to 1.

The stability conditions (1.4) do not depend on the positive (large) parameter $\lambda$. Indeed, by the homogeneity of the polynomials in (1.7), the scaling $(\tau, \xi) \rightarrow(\lambda \tau, \lambda \xi)$ reduces the case $\lambda \neq 1$ to the case $\lambda=1$. 
However, the dependence of the solutions of the Cauchy problem on a large (small) parameter is of significant independent interest, and we will return to this problem later.

In the first part of the present paper we will analyze the stability of pencils of the form

$$
\begin{aligned}
& \mathcal{R}(\tau, \xi):=\mathcal{P}(\tau, \xi)-i \mathcal{Q}(\tau, \xi) \\
& \mathcal{P}(\tau, \xi)=P_{0}(\tau, \xi)+\sum_{j=1}^{\left[\frac{N}{2}\right]}(-1)^{j} \gamma_{2 j} P_{2 j}(\tau, \xi), \\
& \mathcal{Q}(\tau, \xi)=\sum_{j=0}^{\left[\frac{N-1}{2}\right]}(-1)^{j} \gamma_{2 j+1} P_{2 j+1}(\tau, \xi),
\end{aligned}
$$

where $P_{j}(\tau, \xi), j=0, \ldots, N$, are real homogeneous polynomials of degree $m-j$ with

$$
\gamma_{j}>0, \quad P_{j}(1,0)=1, \quad j=1, \ldots, N .
$$

Polynomials of the form (1.12) will be called polynomial pencils of order $(m, N)$.

In principle, Lemma 1.1 implies that for each $\xi \neq 0$ we can apply the Routh-Hurwitz criterion, and for $\xi=0$ we can use this criterion for the polynomials (1.9).

However, is we keep in mind applications to concrete systems occurring in mathematical physics, then the most interesting are stability criteria that can be formulated in terms of the homogeneous polynomials $P_{j}(\tau, \xi)$.

Polynomial pencils of order $(m, 1)$ were studied in 4, where necessary and sufficient conditions for the stability were found. These conditions consist of the requirement that the polynomials $P_{0}(\tau, \xi)$ and $P_{1}(\tau, \xi)$ are strictly hyperbolic and the roots of $P_{1}$ strictly interleave the roots of $P_{0}$. This study was continued in [5], where necessary and sufficient conditions for the stability of the polynomials $P_{0}-i P_{1}-P_{2}$ were found. These conditions are formulated in terms of the roots of the polynomials $P_{0}, P_{1}, P_{2}$. In the case $N=2$ (as opposed to the case $N=1$ ) there are two criteria. The first (weaker) one guarantees the stability. The second (stronger) implies some two-sided estimates that allow us, for differential operators with symbol (1.12), to pass to the case of variable coefficients.

In the case $N>2$ we could not obtain equally complete results. In $\S 4$ we prove necessary stability conditions for arbitrary pencils of order $(m, N)$ (Theorem 4.1) and also very close to them sufficient conditions (Theorem 4.2). A slight strengthening of sufficient conditions (Theorem 4.3) yields a necessary and sufficient condition for the existence of two-sided estimates that are used in the second part of the paper to study equations with a small parameter and variable coefficients. In particular, in $\S 5$ we show that the results of [5] follow from these theorems. The stability conditions are analyzed in more detail in the case $N=3$.

The second part of the paper is devoted to uniform bounds for solutions of the Cauchy problem for strictly hyperbolic partial differential operators with a small

$$
R\left(t, x, \frac{\partial}{\partial t}, \frac{\partial}{\partial x}, \varepsilon\right)=\varepsilon^{N} P_{0}\left(t, x, \frac{\partial}{\partial t}, \frac{\partial}{\partial x}\right)+\sum_{j \geq 1} \varepsilon^{N-j} P_{j}\left(t, x, \frac{\partial}{\partial t}, \frac{\partial}{\partial x}\right)
$$

or with a large

$$
\tilde{R}\left(t, x, \frac{\partial}{\partial t}, \frac{\partial}{\partial x}, \varepsilon\right)=P_{0}\left(t, x, \frac{\partial}{\partial t}, \frac{\partial}{\partial x}\right)+\sum_{j \geq 1} \lambda^{j} P_{j}\left(t, x, \frac{\partial}{\partial t}, \frac{\partial}{\partial x}\right)
$$

parameter. We assume that for each $(t, x) \in \mathbb{R}^{n+1}$ the symbol of the operator (1.13) satisfies the conditions of Theorem 4.3 It turns out that these conditions are necessary and sufficient for the existence of uniform estimates of quadratic forms that are naturally 
associated to the operators (1.13) (or (1.14)). As a corollary of the above estimates, we obtain a uniform (with respect to $\varepsilon$ or $\lambda$ ) bound for the solutions of the Cauchy problem for the operator (1.13) (resp. (1.14)).

As we have already mentioned at the beginning of this introduction, the interest to the questions of stability of pencils of the above form arose in the study of the asymptotic stability of the linearized Grad lattice, which approximates the kinetic Boltzmann equation. Corresponding examples are presented in [5]. For the kinetic Fokker-Planck equation the Grad approach leads to a nice class of hyperbolic equations with constant coefficients. Dispersion equations for these systems are of the form considered in the present paper. The corresponding results are presented in the concluding $\S 9$.

M. M. Malamud read the manuscript of this paper and made a number of useful remarks. The authors express their sincere gratitude to him.

\section{The Hermite-Biehler Theorem and hyperbolic pairs}

The theory of stability of polynomials is based on the classical Hermite-Biehler Theorem. In the first part of this section we will state this theorem in the form that is most convenient for our purposes. In the second part we will formulate the results of [4] on strictly and nonstrictly hyperbolic pairs, which are closely related to the Hermite-Biehler Theorem.

2.1. Remarks on the Hermite-Biehler Theorem. The following formulation of the Hermite-Biehler Theorem is convenient for our purposes.

Theorem 2.1. Let $p(\tau)$ and $q(\tau)$ be polynomials with real coefficients of degree $m$ and $m-1$, respectively, in one variable $\tau$. Then the following conditions are equivalent:

(i) All zeros of the complex polynomial $r(\tau):=p(\tau)-i q(\tau)$ lie in the open half-plane $\operatorname{Im} \tau>0$.

(ii) The leading coefficients of the polynomials $p$ and $q$ have the same sign, and the zeros of the polynomials $p$ and $q$ are real and simple, with zeros of $q$ strictly separating the zeros of $p$.

(iii) All zeros of $p$ are real and simple; denoting them by $p_{1}, \ldots, p_{m}$ we have

$$
\frac{q(\tau)}{p(\tau)}=\sum_{j=1}^{m} \frac{\mu_{j}}{\tau-p_{j}}
$$

where all the numbers $\mu_{j}$ are positive.

(iv) All zeros of $p$ are real and simple and, moreover,

$$
[[p, q]](\tau):=\frac{\operatorname{Im}[p(\tau) \overline{q(\tau)}]}{\operatorname{Im} \tau}>0, \quad \operatorname{Im} \tau \leq 0 .
$$

(iv $)_{0}$ All zeros of $p$ are real and, moreover, for each real $\tau:=\sigma$ we have

$$
[p, q](\sigma):=p^{\prime}(\sigma) q(\sigma)-q^{\prime}(\sigma) p(\sigma)>0, \quad \sigma \in \mathbb{R} .
$$

The equivalence of (i) and (ii) is the classical Hermite-Biehler Theorem; see [6]. The equivalence of (i), (ii) and (iii) is also well known (see, e.g., 7], Lemma 9.1.3]). Therefore, it remains to show the equivalence of conditions (iii), (iv) and (iv $)_{0}$.

Proof. (iii) $\rightarrow$ (iv). By (2.1),

$$
p(\tau) \overline{q(\tau)}=|p(\tau)|^{2}\left(\overline{q(\tau) p^{-1}(\tau)}\right)=|p(\tau)|^{2} \sum_{j=1}^{m} \frac{\mu_{j}}{\bar{\tau}-p_{j}} .
$$


Taking the imaginary part of the expression on the right-hand side and dividing it by $\operatorname{Im} \tau$ we obtain the equality

$$
[[p, q]](\tau)=\sum_{j=1}^{m} \mu_{j} \prod_{k \neq j}\left|\tau-p_{k}\right|^{2}
$$

which implies that the left-hand side depends continuously on $\operatorname{Im} \tau$ and is well defined on the line $\operatorname{Im} \tau=0$. For $\operatorname{Im} \tau<0$ this expression is positive since all roots $p_{k}$ are real and the numbers $\mu_{j}$ are positive. For $\operatorname{Im} \tau=0$ one should additionally note that all roots $p_{j}$ are distinct.

(iv) $\rightarrow\left(\right.$ iv $\left._{0}\right)$. We note that for small $\operatorname{Im} \tau<0$ and $\sigma=\operatorname{Re} \tau$ we have

$$
\operatorname{Im}[p(\tau) q(\bar{\tau})]=\operatorname{Im} \tau\left(p^{\prime}(\sigma) q(\sigma)-q^{\prime}(\sigma) p(\sigma)\right)+O\left(|\operatorname{Im} \tau|^{3}\right) .
$$

Hence

$$
[p, q](\sigma)=\lim _{\rho \rightarrow-0}[[p, q]](\sigma+i \rho)>0
$$

$\left(\mathrm{iv}_{0}\right) \rightarrow($ iii). First of all, by definition $(2.3)$ the function $[p, q](\sigma)$ vanishes at multiple roots of the polynomial $p$. Therefore, (2.3) implies that the polynomial $p$ has distinct real roots. Next,

$$
[p, q](\sigma)=-(p(\sigma))^{2} \frac{d}{d \sigma}\left(\frac{q(\sigma)}{p(\sigma)}\right) .
$$

Since the roots $p_{1}, \ldots, p_{m}$ are real and simple, expanding the fraction $\frac{q(\sigma)}{p(\sigma)}$ into elementary fractions, we can rewrite inequality (2.3) in the form

$$
[p, q](\sigma)=(p(\sigma))^{2} \sum_{j=1}^{m} \frac{\mu_{j}}{\left(\sigma-p_{j}\right)^{2}}=\sum_{j=1}^{m} \mu_{j} \prod_{k \neq j}\left(\sigma-p_{k}\right)^{2}>0 .
$$

Substituting $\sigma=p_{j}$ we obtain

$$
\mu_{j} \prod_{k \neq j}\left(p_{j}-p_{k}\right)^{2}>0
$$

which implies the positivity of the numbers $\mu_{1}, \ldots, \mu_{m}$.

We need one simple (but very important for further applications) corollary of Theorem 2.1. Consider polynomials of the form

$$
r(\tau, \theta)=p(\tau, \theta)-i q(\tau, \theta),
$$

where $p$ and $q$ are real polynomials whose lowest coefficients are continuous functions of $\theta$ varying in a certain domain $\Omega$ :

$$
p(\tau, \theta)=a_{0} \tau^{m}+\sum_{j \geq 1} a_{j}(\theta) \tau^{m-2 j}, \quad q(\tau, \theta)=b_{0} \tau^{m-1}+\sum_{j \geq 1} b_{j}(\theta) \tau^{m-1-2 j} .
$$

Proposition 2.1. Let the following condition (cf. (2.3)) hold for polynomials (2.5):

$$
[p(\cdot, \theta), q(\cdot, \theta)](\sigma)>0 \quad \forall \sigma \in \mathbb{R}, \forall \theta \in \Omega .
$$

Assume additionally that for some $\theta^{0} \in \Omega$ the polynomial $r\left(\tau, \theta^{0}\right)$ is stable. Then the polynomial $r(\tau, \theta)$ is stable for each $\theta$ in the connected component of the point $\theta^{0}$ in $\Omega$.

Proof. Denote by $\tau_{j}(\theta), j=1, \ldots, m$, the roots of the equation $r(\tau, \theta)=0$. Since the leading coefficient of $r$ does not depend on $\theta$ and the remaining coefficients are continuous functions of $\theta$, we can choose $m$ continuous branches $\tau_{j}(\theta)$ of the roots. By the hypothesis of the proposition,

$$
\operatorname{Im} \tau_{j}\left(\theta^{0}\right)>0, \quad j=1, \ldots, m .
$$


Suppose the stability of the polynomials $r(\tau, \theta)$ fails for some $\theta=\theta^{1}$, i.e., for some $j=j_{0}$ we have

$$
\operatorname{Im} \tau_{j_{0}}\left(\theta^{1}\right) \leq 0
$$

Since $\theta^{1}$ belongs to the connected component of $\theta^{0}$ in $\Omega$, there exists a curve $\gamma(s) \in \Omega$, $s_{0} \leq s \leq s_{1}$, such that $\theta^{0}=\gamma\left(s_{0}\right)$ and $\theta^{1}=\gamma\left(s_{1}\right)$. By continuity, there exists $s_{*} \in\left(s_{0}, s_{1}\right)$ such that $\operatorname{Im} \tau_{j 0}\left(\theta^{*}\right)=0$, where $\theta^{*}=\gamma\left(s_{*}\right)$. But then $\tau_{j}\left(\theta^{*}\right)$ is a common real root of the polynomials $p\left(\tau, \theta^{*}\right)$ and $q\left(\tau, \theta^{*}\right)$. The existence of such a root contradicts (2.6). The proposition is proved.

\subsection{Strictly and nonstrictly hyperbolic pairs of polynomials.}

Definition 2.1. We say that homogeneous real polynomials $P(\tau, \xi)$ and $Q(\tau, \xi)$ of degree $m$ and $m-1$, respectively, form a nonstrictly hyperbolic pair if

(a) $P$ and $Q$ are resolved with respect to the highest power of $\tau$ and the corresponding coefficients have the same sign;

(b) the roots $p_{j}(\xi)$ and $q_{j}(\xi)$ of these polynomials are real;

(c) the roots of the polynomial $Q$ separate (nonstrictly) the roots of $P$.

Condition (c) means that after an appropriate renumbering of the roots of $P$ and $Q$ we have

$$
p_{1}(\xi) \geq q_{1}(\xi) \geq \cdots \geq q_{m-1}(\xi) \geq p_{m}(\xi) .
$$

Definition 2.2. A nonstrictly hyperbolic pair $P(\tau, \xi)$ and $Q(\tau, \xi)$ is called strictly hyperbolic if for $\xi \neq 0$ all roots of $P$ and $Q$ are pairwise distinct and inequalities (2.9) are strict.

Strictly and nonstrictly hyperbolic pairs were studied in [4]. Using homogeneity considerations, we can reformulate Theorem 2.1 for strictly hyperbolic pairs in the following way.

We retain notation (2.2) for polynomials in several variables (2.3), setting

$$
\begin{aligned}
{[[P, Q]](\tau, \xi) } & =\frac{\operatorname{Im}(P(\tau, \xi) \overline{Q(\tau, \xi)})}{\operatorname{Im} \tau}, \\
{[P, Q](\sigma, \xi) } & =\partial_{\sigma} P(\sigma, \xi) Q(\sigma, \xi)-\partial_{\sigma} Q(\sigma, \xi) P(\sigma, \xi) .
\end{aligned}
$$

Proposition 2.2. Let $P(\tau, \xi)$ and $Q(\tau, \xi)$ be homogeneous polynomials with real coefficients, of degree $m$ and $m-1$, respectively. Assume that $P$ and $Q$ are resolved with respect to the highest power of $\tau$. Then the following conditions are equivalent:

(i) The polynomial $\mathcal{R}(\tau, \xi):=P(\tau, \xi)-i Q(\tau, \xi)$ is stable, i.e.,

$$
\mathcal{R}(\tau, \xi) \neq 0, \quad \operatorname{Im} \tau \leq 0, \quad|\tau|+|\xi|>0 .
$$

(ii) The polynomials $P(\tau, \xi)$ and $Q(\tau, \xi)$ form a strictly hyperbolic pair.

(iii) All zeros $p_{j}(\xi)$ of the polynomial $P(\tau, \xi)$ are real and distinct for $\xi \neq 0$. Moreover,

$$
\frac{Q(\tau, \xi)}{P(\tau, \xi)}=\sum_{j=1}^{m} \frac{\mu_{j}(\xi)}{\tau-p_{j}(\xi)}
$$

where the functions $\mu_{j}(\xi)$ are homogeneous of degree 0 and positive.

(iv) All zeros of the polynomial $P(\tau, \xi)$ are real and distinct for $\xi \neq 0$ and there exists $\kappa>0$ such that

$$
[[P, Q]](\tau, \xi)>\kappa(|\tau|+|\xi|)^{2 m-2}, \quad \operatorname{Im} \tau \leq 0, \quad(\operatorname{Re} \tau, \xi) \in \mathbb{R}^{n+1} .
$$


(iv $\left.{ }_{0}\right)$ All zeros of the polynomial $P(\tau, \xi)$ are real and there exists $\kappa>0$ such that for real $\tau=: \sigma$ we have

$$
[P, Q](\sigma, \xi)>\kappa(|\sigma|+|\xi|)^{2 m-2}, \quad(\sigma, \xi) \in \mathbb{R}^{n+1} .
$$

Nonstrictly hyperbolic pairs are related to a weakened stability condition. We have (cf. [4]) the following result.

Proposition 2.3. Let $P(\tau, \xi)$ and $Q(\tau, \xi)$ be homogeneous polynomials with real coefficients, of degree $m$ and $m-1$, respectively. Assume that $P$ and $Q$ are resolved with respect to the highest power of $\tau$. Then the following conditions are equivalent.

(i) The polynomial $\mathcal{R}(\tau, \xi):=P(\tau, \xi)-i Q(\tau, \xi)$ is nonstrictly stable, i.e.,

$$
\mathcal{R}(\tau, \xi) \neq 0, \quad \operatorname{Im} \tau<0 .
$$

(ii) For each $\xi^{0} \in \mathbb{R}^{n}$ the polynomials $p(\tau):=P\left(\tau, \xi^{0}\right)$ and $q(\tau)=Q\left(\tau, \xi^{0}\right)$ can be represented in the form

$$
p(\tau)=h(\tau) \tilde{p}(\tau), \quad q(\tau)=h(\tau) \tilde{q}(\tau),
$$

where all roots of the polynomial $h(\tau)$ are real and the polynomials $\tilde{p}(\tau)$ and $\tilde{q}(\tau)$ satisfy equivalent conditions of Theorem [2.1.

(iii) All zeros of the polynomial $P(\tau, \xi)$ are real and

$$
[[P, Q]](\tau, \xi)>0, \quad \operatorname{Im} \tau<0, \quad(\operatorname{Re} \tau, \xi) \in \mathbb{R}^{n+1} .
$$

(iv) All zeros of the polynomial $P(\tau, \xi)$ are real and for real $\tau:=\sigma$ we have

$$
[P, Q](\sigma, \xi) \geq 0, \quad(\sigma, \xi) \in \mathbb{R}^{n+1} .
$$

We note that condition (ii) holds if and only if the polynomials $P(\tau, \xi)$ and $Q(\tau, \xi)$ form a nonstrictly hyperbolic pair.

\section{Notes about the Roots of polynomials $\mathcal{P}$ and $\mathcal{Q}$}

As we have already mentioned in the introduction, our goal is to study stability of the polynomial pencil (1.12) of order $(m, N)$ :

$$
\begin{gathered}
\mathcal{R}(\tau, \xi):=\mathcal{P}(\tau, \xi)-i \mathcal{Q}(\tau, \xi), \\
\mathcal{P}(\tau, \xi)=P_{0}(\tau, \xi)+\sum_{j=1}^{\left[\frac{N}{2}\right]}(-1)^{j} \gamma_{2 j} P_{2 j}(\tau, \xi), \\
\mathcal{Q}(\tau, \xi)=\sum_{j=0}^{\left[\frac{N-1}{2}\right]}(-1)^{j} \gamma_{2 j+1} P_{2 j+1}(\tau, \xi),
\end{gathered}
$$

where $P_{j}(\tau, \xi), j=0, \ldots, N$, are real homogeneous polynomials of degree $m-j$ and

$$
\gamma_{j}>0, \quad P_{j}(1,0)=1, \quad j=1, \ldots, N .
$$

To achieve this goal, we need some information about the relation of the roots of this pencil, as well as of the roots of polynomials (3.2), with the roots of $P_{0}$ and $P_{1}$ (short wave asymptotic occurring as $|\xi| \rightarrow \infty$ ) and with the roots of $P_{N-1}$ and $P_{N}$ (long wave asymptotics occurring as $|\xi| \rightarrow 0$ ). We note also that as $|\xi| \rightarrow 0$, we come across an important notion of boundary layer roots. 
3.1. Asymptotics of the roots of the polynomials $\mathcal{P}$ and $\mathcal{Q}$ for large $|\xi|$. Denote by $p_{j}^{(l)}(\xi)$ the roots of the polynomials $P_{l}(\tau, \xi), l=0,1, \ldots, N$.

Proposition 3.1. We have

$$
p_{j}(\xi)=p_{j}^{(0)}(\xi)+o(|\xi|), \quad q_{j}(\xi)=p_{j}^{(1)}(\xi)+o(|\xi|), \quad|\xi| \rightarrow \infty .
$$

Proof. We write the roots $p_{j}(\xi)$ and $q_{j}(\xi)$ of the polynomials $\mathcal{P}$ and $\mathcal{Q}$ in the form

$$
p_{j}(\xi)=|\xi| \tilde{p}_{j}(\omega,|\xi|), \quad q_{j}(\xi)=|\xi| \tilde{q}_{j}(\omega,|\xi|), \quad \omega=\xi /|\xi| .
$$

To prove the first equality in (3.4) we note that $\tilde{\tau}=\tilde{p}_{j}(\omega,|\xi|)$ are the roots of an algebraic equation

$$
P_{0}(\tilde{\tau}, \omega)-|\xi|^{-2} \gamma_{2} P_{2}(\tilde{\tau}, \omega)+O\left(|\xi|^{-4}\right)=0
$$

and for large $|\xi|$ these roots depend continuously of the small parameter $|\xi|^{-2}$. Moreover,

$$
\tilde{p}_{j}(\omega,|\xi|)=p_{j}^{(0)}(\omega)+O\left(|\xi|^{-2 / r}\right),
$$

where $r$ is the maximum multiplicity of the roots of the polynomial $P_{0}(\tau, \xi)$. This implies the first equality in (3.4). The second equality in (3.4) is proved similarly.

The behavior of the roots of $\mathcal{P}$ and $\mathcal{Q}$ for small $|\xi|$ is a somewhat more delicate question. The description of the asymptotic behavior includes, in addition to the roots of $p_{j}^{(N-1)}(\xi)$ and $p_{j}^{(N)}(\xi)$, the so-called boundary layer roots. The equations for these roots depend on the parity of $N$ and on the polynomials $p(\tau)$ and $q(\tau)$ that occur at $\xi=0$.

3.2. Remarks on the roots of the polynomials $p$ and $q$. According to [2.3] , for $N$ even we have

$$
\begin{aligned}
& \mathcal{P}(\tau, \xi)=P_{0}(\tau, \xi)+\cdots+(-1)^{\frac{N}{2}} \gamma_{N} P_{N}(\tau, \xi) \\
& \mathcal{Q}(\tau, \xi)=\gamma_{1} P_{1}(\tau, \xi)+\cdots+(-1)^{\frac{N}{2}-1} \gamma_{N-1} P_{N-1}(\tau, \xi)
\end{aligned}
$$

For $p(\tau)-i q(\tau):=\mathcal{R}(\tau, 0) \tau^{-(m-N)}$ we obtain the formula

$$
\begin{aligned}
& p(\tau)=\tau^{N}-\gamma_{2} \tau^{N-2}+\cdots+(-1)^{\frac{N}{2}} \gamma_{N}, \\
& q(\tau)=\tau\left(\gamma_{1} \tau^{N-2}-\gamma_{3} \tau^{N-4}+\cdots+(-1)^{\frac{N}{2}-1} \gamma_{N-1}\right) .
\end{aligned}
$$

For $N$ odd we have

$$
\begin{aligned}
& \mathcal{P}(\tau, \xi)=P_{0}(\tau, \xi)+\cdots+(-1)^{\frac{N-1}{2}} \gamma_{N-1} P_{N-1}(\tau, \xi) \\
& \mathcal{Q}(\tau, \xi)=\gamma_{1} P_{1}(\tau, \xi)+\cdots+(-1)^{\frac{N-1}{2}-1} \gamma_{N} P_{N}(\tau, \xi)
\end{aligned}
$$

Accordingly, for $p(\tau)$ and $q(\tau)$ we have

$$
\begin{aligned}
& p(\tau)=\tau\left(\tau^{N-1}-\gamma_{2} \tau^{N-3}+\cdots+(-1)^{\frac{N-1}{2}} \gamma_{N-1}\right), \\
& q(\tau)=\gamma_{1} \tau^{N-1}-\gamma_{3} \tau^{N-3}+\cdots+(-1)^{\frac{N}{2}-1} \gamma_{N} .
\end{aligned}
$$

Lemma 3.1. Let the polynomial $p(\tau)-i q(\tau)$ be stable, so that (the Hermite-Biehler Theorem) $p(\tau)$ and $q(\tau)$ have distinct real roots. Then

(A) For $N$ even the polynomial (3.6) has (real) roots

$$
z_{1}>z_{2}>\cdots>z_{\frac{N}{2}}>z_{\frac{N}{2}+1}=-z_{\frac{N}{2}}>\cdots>z_{N-1}=-z_{1},
$$

the polynomial (3.7) has (real) roots

$$
\zeta_{1}>\zeta_{2}>\cdots>\zeta_{\frac{N}{2}-1}>0>\zeta_{\frac{N}{2}}>\cdots>\zeta_{N-2},
$$


and the roots are interleaving:

$$
\begin{aligned}
z_{1}>\zeta_{1} & >z_{2}>\zeta_{2}>\cdots>z_{\frac{N}{2}-1}>\zeta_{\frac{N}{2}-1}>z_{\frac{N}{2}}>0 \\
& >z_{\frac{N}{2}+1}>\zeta_{\frac{N}{2}}>\cdots>z_{N-1}>\zeta_{N-2}>z_{N} .
\end{aligned}
$$

(B) For $N$ odd the polynomial (3.8) has (real) roots

$$
z_{1}>z_{2}>\cdots>z_{\frac{N-1}{2}}>0>z_{\frac{N+1}{2}}=-z_{\frac{N-1}{2}}>\cdots>z_{N}=-z_{1},
$$

the polynomial (3.9) has (real) roots

$$
\zeta_{1}>\zeta_{2}>\cdots>\zeta_{\frac{N-1}{2}}>\zeta_{\frac{N+1}{2}}>\cdots>\zeta_{N-1},
$$

and the roots are interleaving:

$$
\begin{aligned}
z_{1}>\zeta_{1}>z_{2} & >\zeta_{2}>\cdots>z_{\frac{N-1}{2}}>\zeta_{\frac{N-1}{2}}>0 \\
& >\zeta_{\frac{N+1}{2}}>z_{\frac{N+1}{2}}>\cdots>\zeta_{N-1}>z_{N-1} .
\end{aligned}
$$

Proof. As we have already noted in the statement of the lemma, the roots $z_{1}, \ldots, z_{N}$ of the polynomial (3.6) are real and distinct. Since (3.6) is a polynomial of $\tau^{2}$, together with each root $z_{j}>0$ it also has the root $-z_{j}$. Therefore we can enumerate the roots $z_{1}, \ldots, z_{N}$ so that (3.10) holds.

Similarly, the polynomial $q$ of the form (3.7) has the zero root and the remaining roots are the roots of the second factor, which is a polynomial of $\tau^{2}$. Therefore, we can enumerate the roots $\zeta_{1}, \ldots, 0, \ldots, \zeta_{N-2}$ so that (3.11) holds. (3.7).

Inequalities (3.12) follow from the interleaving of the roots of polynomials (3.6) and

The case of an odd $N$ is treated similarly.

\subsection{The asymptotics of the roots of polynomials $\mathcal{P}$ and $\mathcal{Q}$ for small $|\xi|$.}

Proposition 3.2. Let the conditions of Lemma 3.1 be satisfied and let $z_{1}, \ldots, z_{N}, \zeta_{1}, \ldots$, $\zeta_{N-1}$ be the roots of the polynomials $p(\tau)$ and $q(\tau)$, respectively.

(A) For $N$ odd the roots $p_{1}(\xi), \ldots, p_{m}(\xi)$ of the equation $\mathcal{P}(\tau, \xi)=0$ can be enumerated so that

$$
\begin{aligned}
& p_{j}(\xi)=z_{j}+o(1), \quad|\xi| \rightarrow 0, \quad j=1, \ldots, \frac{N}{2}, \\
& p_{j}(\xi)=z_{j-m+N}+o(1), \quad|\xi| \rightarrow 0, \quad j=m-\frac{N}{2}+1, \ldots, m, \\
& p_{j}(\xi)=p_{j-\frac{N}{2}}^{(N)}(\xi)+o(|\xi|), \quad|\xi| \rightarrow 0, \quad j=\frac{N}{2}+1, \ldots, m-\frac{N}{2} .
\end{aligned}
$$

Similarly, the roots $q_{1}(\xi), \ldots, q_{m-1}(\xi)$ of the equation $\mathcal{Q}(\tau, \xi)=0$ can be enumerated so that

$$
\begin{array}{lll}
q_{j}(\xi)=\zeta_{j}+o(1), & |\xi| \rightarrow 0, & j=1, \ldots, \frac{N}{2}-1 \\
q_{j}(\xi)=\zeta_{j-m+N}+o(1), & |\xi| \rightarrow 0, & j=m-\frac{N}{2}+1, \ldots, m-1 . \\
q_{j}(\xi)=p_{j-\frac{N}{2}+1}^{(N-1)}(\xi)+o(|\xi|), & |\xi| \rightarrow 0, & j=\frac{N}{2}, \ldots, m-\frac{N}{2} .
\end{array}
$$


(B) For $N$ odd the roots $p_{1}(\xi), \ldots, p_{m}(\xi)$ of the equation $\mathcal{P}(\tau, \xi)=0$ can be enumerated so that

$$
\begin{array}{lll}
p_{j}(\xi)=z_{j}+o(1), & |\xi| \rightarrow 0, & j=1, \ldots, \frac{N-1}{2}, \\
p_{j}(\xi)=z_{j-m+N}+o(1), & |\xi| \rightarrow 0, & j=m-\frac{N+1}{2}, \ldots, m, \\
p_{j}(\xi)=p_{j-\frac{N-1}{2}}^{(N-1)}(\xi)+o(|\xi|), & |\xi| \rightarrow 0, & j=\frac{N+1}{2}, \ldots, m-\frac{N-1}{2} .
\end{array}
$$

Similarly, the roots $q_{1}(\xi), \ldots, q_{m-1}(\xi)$ of the equation $\mathcal{Q}(\tau, \xi)=0$ can be enumerated so that

$$
\begin{aligned}
& q_{j}(\xi)=\zeta_{j}+o(1), \quad|\xi| \rightarrow 0, \quad j=1, \ldots, \frac{N-1}{2}, \\
& q_{j}(\xi)=\zeta_{j-m+N}+o(1), \quad|\xi| \rightarrow 0, \quad j=m-\frac{N-1}{2}, \ldots, m-1, \\
& q_{j}(\xi)=p_{j-\frac{N}{2}+1}^{(N)}(\xi)+o(|\xi|), \quad|\xi| \rightarrow 0, \quad j=\frac{N+1}{2}, \ldots, m-\frac{N+1}{2} .
\end{aligned}
$$

Proof. We prove part (A) since (B) is proved similarly.

As in Proposition 3.1, we make the substitution (3.5) and note that $\tilde{\tau}=\tilde{p}_{j}(\omega,|\xi|)$ and $\tilde{\tau}=\tilde{q}_{j}(\omega,|\xi|)$ are the roots of the algebraic equations

$$
\begin{array}{r}
(-1)^{\frac{N}{2}} \gamma_{N} P_{N}(\tilde{\tau}, \omega)+\sum_{j=0}^{\frac{N}{2}-1}(-1)^{j}|\xi|^{N-2 j} \gamma_{2 j} P_{2 j}(\tilde{\tau}, \omega)=0, \\
(-1)^{\frac{N}{2}-1} \gamma_{N-1} P_{N-1}(\tilde{\tau}, \omega)+\sum_{j=1}^{\frac{N}{2}-1}(-1)^{j}|\xi|^{N-2 j-1} \gamma_{2 j+1} P_{2 j+1}(\tilde{\tau}, \omega)=0,
\end{array}
$$

where we formally put $\gamma_{0}=1$. Using standard arguments of the theory of small perturbations of roots of algebraic equations (see, e.g., [8]) one can show that the first equation in (3.17) has $m-N$ roots, which we denote $\tilde{p}_{\frac{N}{2}+j}(\omega,|\xi|), j=1, \ldots, m-N$; as $|\xi| \rightarrow 0$, these roots tend to the roots $p_{j}^{(N)}(\omega)$ of the limiting equation $P_{N}(\tilde{\tau}, \omega)=0$ obtained by taking $|\xi|=0$. Returning to the variable $\tau=|\xi| \tilde{\tau}$, we construct the family of roots (3.14). Similarly, using the second equation in (3.17), we construct the family of roots (3.16).

As opposed to the roots (3.14), (3.16), the roots of the form (3.13), (3.15) do not tend to zero as $|\xi| \rightarrow 0$; these roots are called boundary layer roots. To construct these roots for $N$ even we expand the polynomial $\mathcal{P}(\tau, \xi)$ in powers of $\xi$ and rewrite the equation $\mathcal{P}(\tau, \xi)=0$ in the form

$$
\tau^{m-N} p(\tau)+\sum_{|\alpha| \geq 1} p_{\alpha}(\tau) \xi^{\alpha}=0
$$

For $\xi=0$ this equation has the roots $z_{1}, \ldots, z_{N}$, which are the roots of the polynomial $p(\tau)$. The theory of small perturbations yields the roots (3.13). Similarly, the boundary layer roots of the equation $\mathcal{Q}(\tau, \xi)=0$ are constructed as small perturbations of the roots of the polynomial $q(\tau)$.

The case of odd $N$ is treated similarly.

\section{Stable hyperbolic Pencils}

4.1. Main stability conditions. In this subsection we present a necessary and a (close to it) sufficient condition of stability for pencils of the general form. 
Theorem 4.1. Let the pencil (3.1) satisfy the stability condition

$$
\mathcal{R}(\tau, \xi) \neq 0, \quad \operatorname{Im} \tau \leq 0, \quad|\tau|+|\xi|>0 .
$$

Then the following holds.

(I) The polynomial $r(\tau):=\frac{\mathcal{R}(\tau, 0)}{\tau^{m-N}}$ in one variable is stable.

(II) The polynomials $P_{N-1}(\tau, \xi)$ and $P_{N}(\tau, \xi)$ form a nonstrictly hyperbolic pair.

(III) The polynomials $P_{0}(\tau, \xi)$ and $P_{1}(\tau, \xi)$ form a nonstrictly hyperbolic pair.

(IV) The condition

$$
[[\mathcal{P}, \mathcal{Q}]](\tau, \xi)>0, \quad \operatorname{Im} \tau \leq 0, \quad|\tau|+|\xi|>0
$$

holds.

Proof. Condition (I) immediately follows from (4.1). The proof of the remaining conditions is based on Theorem 2.1

First, note that condition (IV) follows directly from this theorem.

Next, by Theorem 2.1 the roots of the polynomials $p_{j}(\xi)$ and $q_{j}(\xi)$ are real and distinct, and with an appropriate enumeration the roots of the second polynomial strictly interleave the roots of the first polynomial. To deduce from this fact the information about the roots of the pairs $P_{0}, P_{1}$ and $P_{N-1}, P_{N}$ respectively, we need to use short wave or long wave asymptotics of the roots.

Proof of Condition (III). According to (3.4), the fact that the roots of $p_{j}(\xi)$ and $q_{j}(\xi)$ are real implies that the roots of the polynomials $P_{0}$ and $P_{1}$ are real as well (although, in general, multiple roots can appear). Formula (3.4) and strict interleaving of the roots of $p_{j}(\xi)$ and $q_{j}(\xi)$ imply nonstrict interleaving of the roots of $P_{0}$ and $P_{1}$.

Proof of Condition (II) is similar, but one should use Proposition 3.2. We note that the roots of the polynomials $P_{N-1}(\tau, \xi)$ and $P_{N}(\tau, \xi)$, and converging to them roots of $p_{j}(\xi)$ and $q_{j}(\xi)$ respectively, are of order $O(|\xi|)$ as $|\xi| \rightarrow 0$, contrary to the boundary layer roots, which are of order $O(1)$. Therefore, the interleaving of the roots of $\mathcal{P}$ and $\mathcal{Q}$ implies the interleaving of the roots of $P_{N-1}$ and $P_{N}$.

The theorem is proved.

Now we pass to sufficient conditions for stability of hyperbolic pencils of the general form. We will assume that conditions 1 and 2 formulated in the Introduction hold. Hence,

1. The polynomials $P_{j}(\tau, \xi), j=0, \ldots, N$, are real and homogeneous, with the homogeneity degree of $P_{j}$ equal to $m-j$.

2. The polynomials $P_{j}, j=0, \ldots, N$, are resolved with respect to the highest power of $\tau$.

A partial converse to Theorem 4.1 is the following result.

Theorem 4.2. Let polynomials $P_{j}(\tau, \xi)$ satisfy conditions 1 and 2. Also let conditions (I), (II), (III) of Theorem 4.1 hold and

$\left(\mathrm{IV}_{0}\right)[\mathcal{P}, \mathcal{Q}](\sigma, \xi)>0 \quad \forall(\sigma, \xi) \in \mathbb{R}^{n+1} \backslash\{0\}$.

Additionally, let one of the following two conditions be satisfied.

( $\left.\mathrm{II}_{0}\right)$ The polynomials $P_{N-1}(\tau, \xi)$ and $P_{N}(\tau, \xi)$ form a strictly hyperbolic pair.

(III $)$ The polynomials $P_{0}(\tau, \xi)$ and $P_{1}(\tau, \xi)$ form a strictly hyperbolic pair.

Then the stability condition (4.1) holds.

Proof. We argue according to the following plan. From $\left(\mathrm{II}_{0}\right)$ we deduce that for sufficiently small nonzero $\xi$ the polynomials $\mathcal{P}(\tau, \xi)$ and $\mathcal{Q}(\tau, \xi)$ have distinct real roots and the roots of $\mathcal{Q}$ interleave the roots of $\mathcal{P}$. By Theorem [2.1, for such $\xi$ the polynomial $\mathcal{P}-i \mathcal{Q}$ is stable. Using condition $\left(\mathrm{IV}_{0}\right)$ and Proposition [2.1] we prove (4.1). 
Similarly, condition $\left(\mathrm{III}_{0}\right)$ implies the stability of $\mathcal{P}-i \mathcal{Q}$ for sufficiently large $|\xi|$, after which we again use $\left(\mathrm{IV}_{0}\right)$ and Proposition 2.1.

Now we pass to detailed argument. We start with the technically simpler case where $\left(\mathrm{III}_{0}\right)$ holds. In proving Proposition 3.1 we noted that for sufficiently large $|\xi|$ and each fixed $\omega=\frac{\xi}{|\xi|}$ the number $\tilde{\sigma}=\tilde{p}_{j}(\omega,|\xi|)=\frac{p_{j}(\xi)}{|\xi|}$ associated to the $\operatorname{root} p_{j}(\xi)$ is a small perturbation of a root $p_{j}^{0}(\xi)$ of the limiting equation $P_{0}(\tilde{\sigma}, \omega)=0$. Moreover, $\tilde{\sigma}$ is a small real perturbation of the root $p_{j}^{0}(\xi)$.

Indeed, $\tilde{\sigma}$ satisfies the following equation with real coefficients:

$$
P_{0}(\tilde{\sigma}, \omega)-|\xi|^{-2} H(\tilde{\sigma}, \omega,|\xi|)=0,
$$

where we denote for brevity

$$
H(\tilde{\sigma}, \omega,|\xi|):=\gamma_{2} P_{2}(\tilde{\sigma}, \omega)-|\xi|^{-2} \gamma_{4} P_{4}(\tilde{\sigma}, \omega)+\cdots=\sum_{k=0} h_{k}(\tilde{\sigma}, \omega)|\xi|^{-2 k-2} .
$$

We look for the solution of (4.2) in the form of a series in powers of $|\xi|^{-2}$ :

$$
\tilde{\sigma}=p_{j}^{0}(\omega)+\sum_{k=1}^{\infty} a_{k}(\omega)|\xi|^{-2 k} .
$$

Substituting this series in (4.2), we obtain recurrence relations for the coefficients $a_{k}(\omega)$ :

$$
\begin{aligned}
& \frac{\partial P_{0}}{\partial \tau}\left(p_{j}^{0}(\omega), \omega\right) a_{1}(\omega)=h_{0}\left(p_{j}^{0}(\omega), \omega\right), \\
& \frac{\partial P_{0}}{\partial \tau}\left(p_{j}^{0}(\omega), \omega\right) a_{2}(\omega)=-a_{1}^{2}(\omega) \frac{\partial^{2} P_{0}}{\partial \tau^{2}}\left(p_{j}^{0}(\omega), \omega\right)+a_{1}(\omega) \frac{\partial h_{0}}{\partial \tau}\left(p_{j}^{0}(\omega), \omega\right) .
\end{aligned}
$$

Continuing further, we obtain an equation for an arbitrary $a_{k}$, whose left-hand side is of the form

$$
\frac{\partial P_{0}}{\partial \tau}\left(p_{j}^{0}(\omega), \omega\right) a_{k}(\omega)
$$

and the right-hand side contains a polynomial in $a_{1}, \ldots, a_{k-1}$ with coefficients equal to the derivatives of the polynomials $P_{0}, P_{2}, \ldots$ at the point $\left(p_{j}^{0}(\omega), \omega\right)$. Therefore, we consecutively determine $a_{k}$ from certain real linear equations.

By strict hyperbolicity of $P_{0}(\sigma, \omega)$, the absolute value of the coefficient on the lefthand side is bounded below by a positive constant, so that we can resolve all recurrence equations and determine real coefficients of the series (4.3).

Therefore, strict hyperbolicity of $P_{0}$ implies that the roots $p_{j}$ of the polynomial $\mathcal{P}$ are real. By (3.4), for sufficiently large $|\xi|$ these roots are distinct. Similarly, strict hyperbolicity of $P_{1}$ implies that the roots $q_{j}$ of the polynomial $\mathcal{Q}$ are real and distinct. Since the roots $p_{j}^{1}(\omega)$ separate the roots $p_{j}^{0}(\omega)$, asymptotic formulas (3.4) imply that the roots of $\mathcal{Q}$ separate the roots of $\mathcal{P}$.

Using similar (but more cumbersome) arguments based on Proposition 3.2, the conclusion of the theorem can be derived from $\left(\mathrm{II}_{0}\right)$.

The theorem is proved.

4.2. Estimates for imaginary and real parts of the polynomial $\mathcal{R}$. In the case where conditions $\left(\mathrm{II}_{0}\right)$ and $\left(\mathrm{III}_{0}\right)$ hold simultaneously, the conclusion of Theorem $4.2 \mathrm{can}$ be made stronger. We have the following result.

Theorem 4.3. Let a polynomial $P_{j}(\tau, \xi)$ satisfy conditions 1 and 2 in the Introduction. Then the following statements are equivalent.

(A) Conditions (I), $\left(\mathrm{II}_{0}\right),\left(\mathrm{III}_{0}\right)$ and $\left(\mathrm{IV}_{0}\right)$ of Theorem 4.2 hold. 
(B) The polynomials $\mathcal{P}(\sigma, \xi)$ and $\mathcal{Q}(\sigma, \xi)$ have no common nonzero real roots and the estimate

$$
\begin{aligned}
& -\operatorname{Im}(\mathcal{P}(\tau, \xi) \overline{\mathcal{Q}(\tau, \xi)}) \\
& \geq C|\operatorname{Im} \tau|\left((|\tau|+|\xi|)^{2 m-2}+(|\tau|+|\xi|)^{2 m-4}+\cdots+(|\tau|+|\xi|)^{2 m-2 N}\right) \\
& \quad \operatorname{Im} \tau \leq 0, \quad(\operatorname{Re} \tau, \xi) \in \mathbb{R}^{n+1}
\end{aligned}
$$

holds.

Proof. (A) $\rightarrow$ (B). First of all, note that condition (A) implies all assumptions of Theorem 4.2, so that the polynomial $\mathcal{R}$ is stable. Therefore, the polynomials $\mathcal{P}(\sigma, \xi)$ and $\mathcal{Q}(\sigma, \xi)$ have no common nonzero real roots. Next, by Theorem 4.1, the stronger (compared to $\left(\mathrm{IV}_{0}\right)$ ) condition (IV) holds.

We note that according to (2.11) we have

$$
\begin{aligned}
& -\operatorname{Im}(\mathcal{P}(\tau, \xi) \overline{\mathcal{Q}(\tau, \xi)})=-\operatorname{Im} \tau[[\mathcal{P}, \mathcal{Q}]](\tau, \xi) \\
& =-\operatorname{Im} \tau \sum_{i=0}^{\left[\frac{N}{2}\right]} \sum_{j=0}^{\left[\frac{N-1}{2}\right]}(-1)^{i+j} \gamma_{2 i} \gamma_{2 j+1}\left[\left[P_{2 i}, P_{2 j+1}\right]\right](\tau, \xi),
\end{aligned}
$$

and for $\operatorname{Im} \tau \leq 0$ the inequality (4.4) is reduced to the following inequality:

$$
\begin{aligned}
|[\mathcal{P}, \mathcal{Q}]|(\tau, \xi) & =\sum_{i=0}^{\left[\frac{N}{2}\right]} \sum_{j=0}^{\left[\frac{N-1}{2}\right]}(-1)^{i+j} \gamma_{2 i} \gamma_{2 j+1}\left[\left[P_{2 i}, P_{2 j+1}\right]\right](\tau, \xi) \\
& \geq C\left((|\tau|+|\xi|)^{2 m-2}+(|\tau|+|\xi|)^{2 m-4}+\cdots+(|\tau|+|\xi|)^{2 m-2 N-2}\right) .
\end{aligned}
$$

On the compact set $\delta \leq|\tau|+|\xi| \leq \delta^{-1}$ the inequality (4.5) holds by condition (IV) (of course, with a constant depending on $\delta$ ).

In proving (4.5) for large and for small $|\xi|$ one must analyze the sum in (4.5) more carefully.

We note that $\left[\left[P_{2 i}, P_{2 j+1}\right]\right](\tau, \xi)$ is a homogeneous polynomial in $(\tau, \xi)$ of degree $2 m-$ $2 i-2 j-2$. The leading term in the sum (4.5) is the polynomial

$$
\gamma_{1}\left[\left[P_{0}, P_{1}\right]\right](\tau, \xi)
$$

of degree $2 m-2$. The polynomial of the smallest degree is

$$
(-1)^{\left[\frac{N}{2}\right]+\left[\frac{N-1}{2}\right]} \gamma_{2\left[\frac{N}{2}\right]} \gamma_{2\left[\frac{N-1}{2}\right]+1}\left[\left[P_{2\left[\frac{N}{2}\right]}, P_{2\left[\frac{N-1}{2}\right]+1}\right]\right](\tau, \xi) .
$$

For $N$ even we have

$$
2\left[\frac{N}{2}\right]=N, \quad 2\left[\frac{N-1}{2}\right]+1=N-1, \quad\left[\frac{N}{2}\right]+\left[\frac{N-1}{2}\right]=N-1,
$$

and (4.6) equals

$$
-\gamma_{N-1} \gamma_{N}\left[\left[P_{N}, P_{N-1}\right]\right]=\gamma_{N-1} \gamma_{N}\left[\left[P_{N-1}, P_{N}\right]\right] .
$$

For $N$ odd we have

$$
2\left[\frac{N}{2}\right]=N-1, \quad 2\left[\frac{N-1}{2}\right]+1=N, \quad\left[\frac{N}{2}\right]+\left[\frac{N-1}{2}\right]=N-1,
$$

and the expression (4.6) coincides with the right-hand side of (4.7). 
Therefore, we see that

$$
\begin{array}{ll}
{[[\mathcal{P}, \mathcal{Q}]](\tau, \xi)=\gamma_{1}\left[\left[P_{0}, P_{1}\right]\right](\tau, \xi)+O\left((|\tau|+|\xi|)^{2 m-4}\right),} & |\xi| \rightarrow \infty \\
{[[\mathcal{P}, \mathcal{Q}]](\tau, \xi)=\gamma_{N-1} \gamma_{N}\left[\left[P_{N-1}, P_{N}\right]\right](\tau, \xi)+O\left((|\tau|+|\xi|)^{2 m-2 N+2}\right),} & |\xi| \rightarrow 0 .
\end{array}
$$

By $\left(\mathrm{III}_{0}\right)$ and Proposition 2.2. the first term on the right-hand side of (4.8) is bounded below by

$$
\gamma_{1} \kappa(|\tau|+|\xi|)^{2 m-2}
$$

and for $|\tau|+|\xi| \geq \delta^{-1}$ with a sufficiently small $\delta$ the sum in (4.5) is bounded below by the right-hand side of (4.5).

Similarly, $\left(\mathrm{II}_{0}\right)$ implies that the first term on the right-hand side of (4.9) is bounded below by

$$
\gamma_{N-1} \gamma_{N} \kappa(|\tau|+|\xi|)^{2 m-2 N}
$$

and for $|\tau|+|\xi| \leq \delta$ with a sufficiently small $\delta$ the sum in (4.5) is bounded below by the right-hand side of (4.5)).

$(\mathrm{B}) \rightarrow(\mathrm{A})$. By the assumptions, the polynomial $\mathcal{R}(\sigma, \xi)$ takes nonzero values at $|\sigma|+$ $|\xi|>0$. The estimate (4.4) shows that the polynomial $\mathcal{R}(\tau, \xi)$ takes nonzero values at $\operatorname{Im} \tau<0$. Therefore, the polynomial $\mathcal{R}(\tau, \xi)$ is stable. By Theorem 4.1 conditions (I), (II), (III), and (IV) are satisfied. It remains to show that the stronger conditions $\left(\mathrm{II}_{0}\right)$ and $\left(\mathrm{III}_{0}\right)$ are satisfied as well.

To this end, making the substitution $(\tau, \xi) \rightarrow(\rho \tau, \rho \xi)$ in the estimate (4.4), dividing both sides of (4.4) by $\rho^{2 m-1}$ and passing to the limit as $\rho \rightarrow \infty$ we obtain

$$
-\operatorname{Im}\left(P_{0}(\tau, \xi) \overline{P_{1}(\tau, \xi)}\right) \geq C|\operatorname{Im} \tau|(|\tau|+|\xi|)^{2 m-2}, \quad \operatorname{Im} \tau \leq 0 .
$$

Using Proposition 2.2, we prove $\left(\mathrm{III}_{0}\right)$.

On the other hand, dividing the inequality obtained by the above substitution by $\rho^{2 m-2 N+1}$ and passing to the limit as $\rho \rightarrow 0$, we obtain

$$
-\operatorname{Im}\left(P_{N-1}(\tau, \xi) \overline{P_{N}(\tau, \xi)}\right) \geq C|\operatorname{Im} \tau|(|\tau|+|\xi|)^{2 m-2 N}, \quad \operatorname{Im} \tau \leq 0 .
$$

Using Proposition 2.2, we prove $\left(\mathrm{II}_{0}\right)$.

The theorem is proved.

Remark 4.1. Changing the constant in (4.4) we can replace the right-hand side of (4.4) with

$$
|\operatorname{Im} \tau|(|\tau|+|\xi|)^{2 m-2 N}(1+|\tau|+|\xi|)^{2 N-2} .
$$

One can easily see that given the structure of polynomials $\mathcal{P}$ and $\mathcal{Q}$, the estimate will be two-sided, i.e.,

$$
\begin{aligned}
& C^{-1}|\operatorname{Im} \tau|(|\tau|+|\xi|)^{2 m-2 N}(1+|\tau|+|\xi|)^{2 N-2} \\
& \quad \leq-\operatorname{Im} \mathcal{P}(\tau, \xi) \overline{\mathcal{Q}(\tau, \xi)} \leq C|\operatorname{Im} \tau|(|\tau|+|\xi|)^{2 m-2 N}(1+|\tau|+|\xi|)^{2 N-2}
\end{aligned}
$$

4.3. Further analysis of the stability criteria. Now we analyze in more detail condition $\left(\mathrm{IV}_{0}\right)$ of Theorem 4.2. Recall that on the left-hand side of this condition we have the expression

$$
[\mathcal{P}, \mathcal{Q}](\eta)=\sum_{i=0}^{\left[\frac{N}{2}\right]} \sum_{j=0}^{\left[\frac{N-1}{2}\right]}(-1)^{i+j} \gamma_{2 i} \gamma_{2 j+1}\left[P_{2 i}, P_{2 j+1}\right](\eta),
$$

where we set $\eta=(\sigma, \xi)$ and $\gamma_{0}=1$. 
Pass to polar coordinates setting $\eta=\Lambda \tilde{\eta}, \Lambda=|\eta|,|\tilde{\eta}|=1$. Then (4.11) can be rewritten as a polynomial in $\Lambda$ with coefficients depending on $\tilde{\eta}$ :

$$
[\mathcal{P}, \mathcal{Q}](\eta)=a_{0}(\tilde{\eta}) \Lambda^{2 m-2}+\sum_{k=1}^{N-1} a_{k}(\tilde{\eta}) \Lambda^{2 m-2-2 k}+a_{N}(\tilde{\eta}) \Lambda^{2 m-2-2 N},
$$

where

$$
a_{0}(\tilde{\eta})=\gamma_{1}\left[P_{0}, P_{1}\right](\tilde{\eta}), \quad a_{N}(\tilde{\eta})=\gamma_{N-1} \gamma_{N}\left[P_{N-1}, P_{N}\right](\tilde{\eta}) .
$$

For $k=1, \ldots, N-1$ we have

$$
a_{k}(\tilde{\eta})=(-1)^{k} \sum_{i+j=k} \gamma_{2 i} \gamma_{2 j+1}\left[P_{2 i}, P_{2 j+1}\right](\tilde{\eta}) .
$$

We mention special cases of (4.11) (which can also be easily rewritten in the form (4.12)):

1. $N=2$,

$$
[\mathcal{P}, \mathcal{Q}]=\gamma_{1}\left[P_{0}, P_{1}\right]+\gamma_{1} \gamma_{2}\left[P_{1}, P_{2}\right]
$$

2. $N=3$,

$$
[\mathcal{P}, \mathcal{Q}]=\gamma_{1}\left[P_{0}, P_{1}\right]+\left(\gamma_{1} \gamma_{2}\left[P_{1}, P_{2}\right]-\gamma_{3}\left[P_{0}, P_{3}\right]\right)+\gamma_{2} \gamma_{3}\left[P_{2}, P_{3}\right] .
$$

3. $N=4$,

$$
\begin{aligned}
{[\mathcal{P}, \mathcal{Q}]=} & \gamma_{1}\left[P_{0}, P_{1}\right]+\left(\gamma_{1} \gamma_{2}\left[P_{1}, P_{2}\right]-\gamma_{3}\left[P_{0}, P_{3}\right]\right) \\
& +\left(\gamma_{2} \gamma_{3}\left[P_{2}, P_{3}\right]-\gamma_{1} \gamma_{4}\left[P_{1}, P_{4}\right]\right)+\gamma_{3} \gamma_{4}\left[P_{3}, P_{4}\right]
\end{aligned}
$$

To the expression (4.12) we associate the following polynomial of degree $N$ in $Z:=\Lambda^{2}$ with coefficients depending on $\tilde{\eta}$ :

$$
\Delta_{N}(\tilde{\eta}, Z):=a_{0}(\tilde{\eta}) Z^{N}+a_{1}(\tilde{\eta}) Z^{N-1}+\cdots+a_{N-1}(\tilde{\eta}) Z+a_{N}(\tilde{\eta}) .
$$

If the conditions of Theorem 4.3 are satisfied, then $a_{0}(\tilde{\eta})>0, a_{N}(\tilde{\eta})>0$, and the polynomial (4.13) is positive for sufficiently large and sufficiently small $Z$. This implies the existence of a minimum of the polynomial (4.13) as $Z \geq 0,|\tilde{\eta}|=1$. Therefore, condition $\left(\mathrm{IV}_{0}\right)$ can be rewritten in the form

$$
\min _{Z \geq 0,|\tilde{\eta}|=1} \Delta_{N}(\tilde{\eta}, Z)>\delta>0
$$

In verifying this condition one should consider the derivative of the polynomial (4.13):

$$
\Delta_{N}^{\prime}(\tilde{\eta}, Z):=N a_{0}(\tilde{\eta}) Z^{N-1}+\cdots+a_{N-1}(\tilde{\eta}) .
$$

Denote by $Z_{l}(\tilde{\eta}), l=1, \ldots, k$, the real nonnegative roots of the polynomial 4.15). Condition (4.4) is reduced to the following set of conditions:

$$
\min _{|\tilde{\eta}|=1} \Delta_{N}\left(\tilde{\eta}, Z_{l}(\tilde{\eta})\right)>\delta>0, \quad l=1, \ldots, k .
$$

Remark 4.2. If for some $\tilde{\eta}=\tilde{\eta}^{0}$ the polynomial (4.15) has no real nonnegative roots, then the polynomial (4.14) is monotone increasing on the half-line $\{Z \geq 0\}, \tilde{\eta}=\tilde{\eta}^{0}$, and takes the (positive) minimum value at $Z=0$.

\section{Stability CONDITIONS FOR TRIPLES AND QUADRUPLES OF HYPERBOLIC POLYNOMIALS}

In this section we comment on some conditions of the previous section in the cases $N=2$ (which was considered in details in [5]) and $N=3$. 
5.1. Hyperbolic triples. Here we specify the conditions of Theorems 4.14 .3 for pencils of the form

$$
\mathcal{R}(\tau, \xi):=\mathcal{P}(\tau, \xi)-i \mathcal{Q}(\tau, \xi)=P_{0}(\tau, \xi)-P_{2}(\tau, \xi)-i P_{1}(\tau, \xi) .
$$

In this case condition (I) of Theorem 4.1 means that the numbers $P_{0}(1,0), P_{1}(1,0)$, and $P_{2}(1,0)$ are all nonzero and have the same sign. But then we can take this into account in conditions (II) and (III), rewriting them as follows:

(i) $P_{0}(\tau, \xi)$ and $P_{1}(\tau, \xi)$ form a nonstrictly hyperbolic pair.

(ii) $P_{1}(\tau, \xi)$ and $P_{2}(\tau, \xi)$ form a nonstrictly hyperbolic pair.

We now turn to condition (IV). In the case of the polynomial (5.1) it means that

$$
\left[\left[P_{0}, P_{1}\right]\right](\tau, \xi)+\left[\left[P_{1}, P_{2}\right]\right](\tau, \xi)>0, \quad \operatorname{Im} \tau \leq 0 \quad \forall(\operatorname{Re} \tau, \xi) \in \mathbb{R}^{n+1} .
$$

By (i), (ii), and results of 2.2 , each term on the left-hand side is strictly positive for $\operatorname{Im} \tau<0$ and nonnegative for $\operatorname{Im} \tau=0$. Therefore, our condition takes the following form:

(iii) There is no nonzero real vector $\left(\sigma^{0}, \xi^{0}\right)$ such that

$$
\left[P_{0}, P_{1}\right]\left(\sigma^{0}, \xi^{0}\right)=\left[P_{1}, P_{2}\right]\left(\sigma^{0}, \xi^{0}\right)=0 .
$$

Remark 5.1. We mention some consequences of conditions (i)-(iii). First we note that the bracket $\left[P_{i}, P_{j}\right]\left(\sigma^{0}, \xi^{0}\right)$ vanishes on the common zeros of the polynomials $P_{i}$ and $P_{j}$. Therefore, condition (iii) implies the condition

(iii 1 ) There is no nonzero real vector $\left(\sigma^{0}, \xi^{0}\right)$ such that

$$
P_{0}\left(\sigma^{0}, \xi^{0}\right)=P_{1}\left(\sigma^{0}, \xi^{0}\right)=P_{2}\left(\sigma^{0}, \xi^{0}\right)=0 .
$$

Next, if $\left(\sigma^{0}, \xi^{0}\right)$ is a multiple root of the polynomial $P_{1}$, then, by (i) and (ii), this vector is a root of both the polynomial $P_{0}$ and the polynomial $P_{2}$, which contradicts condition ( iii $\left._{1}\right)$. Therefore, we have the condition

(iii $\left.{ }_{2}\right)$ The polynomial $P_{1}(\tau, \xi)$ has simple real roots, i.e., it is strictly hyperbolic.

We note that since $P_{1}=\mathcal{Q}$, condition ( iii $_{2}$ ) is a direct consequence of Theorem 2.1

In [5] it was noted that conditions (i), (ii), and ( $\mathrm{iii}_{2}$ ) are both necessary and sufficient for the stability of the polynomial $\mathcal{R}$. Therefore, we have the following result.

Theorem 5.1. Conditions (i), (ii), (iii) are necessary and sufficient for the stability of the polynomial (5.1).

The necessity is already proved. For completeness, we sketch the proof of sufficiency. For $\operatorname{Im} r \leq 0$ we have

$$
\left.-\operatorname{Im}(\mathcal{R}(\tau, \xi) \overline{\mathcal{Q}(\tau, \xi)})=|\operatorname{Im} \tau|\left[\left[P_{0}, P_{1}\right]\right](\tau, \xi)+|\operatorname{Im} \tau| \mid\left[P_{1}, P_{2}\right]\right](\tau, \xi)+\left|P_{1}(\tau, \xi)\right|^{2} .
$$

By conditions (i), (ii), all three terms on the right-hand side are positive for $\operatorname{Im} \tau<0$. But then the polynomial $\mathcal{R}(\tau, \xi)$ cannot vanish in this (open) half-plane. To prove the stability it remains to show that the polynomial $\mathcal{R}(\tau, \xi)$ does not have nonzero real roots $\left(\sigma^{0}, \xi^{0}\right)$. The existence of such a root would mean that

$$
P_{0}\left(\sigma^{0}, \xi^{0}\right)-P_{2}\left(\sigma^{0}, \xi^{0}\right)=0, \quad P_{1}\left(\sigma^{0}, \xi^{0}\right)=0 .
$$

By condition ( iii $_{1}$ ) (which follows from (iii)) the terms in the difference on the left-hand side cannot vanish simultaneously. Therefore, for (5.2) to hold, it is necessary that the terms in the difference have the same sign. This contradicts the condition of the interleaving of roots of $P_{0}(\sigma, \xi)$ and $P_{1}(\sigma, \xi)$ and, consequently, of $P_{1}(\sigma, \xi)$ and $P_{2}(\sigma, \xi)$ (see [5] for more details). 
Now we turn to Theorem 4.3 Conditions (I) and (IV) follow from conditions $\left(\mathrm{II}_{0}\right)$ and $\left(\mathrm{III}_{0}\right)$. As for the estimate (4.4), it turns out to be equivalent to conditions $\left(\mathrm{II}_{0}\right)$ and $\left(\mathrm{III}_{0}\right)$. Namely, we have the following result (cf. [5]).

Theorem 5.2. The following conditions on polynomials $P_{j}(\tau, \xi), j=0,1,2$, are equivalent.

(A) The polynomials $P_{0}(\tau, \xi), P_{1}(\tau, \xi)$, and $P_{2}(\tau, \xi)$ form a strictly hyperbolic triple in the sense of [5] (i.e., $P_{0}, P_{1}$, and $P_{1}, P_{2}$ are strictly hyperbolic pairs).

(B) We have

$$
\begin{aligned}
& -\operatorname{Im}\left(P_{0}(\tau, \xi)-i P_{1}(\tau, \xi)-P_{2}(\tau, \xi)\right) \overline{P_{1}(\tau, \xi)} \\
& \quad \geq \text { const }|\operatorname{Im} \tau|\left((|\tau|+|\xi|)^{2 m-2}+(|\tau|+|\xi|)^{2 m-4}\right)+\left|P_{1}(\tau, \xi)\right|^{2}, \quad \operatorname{Im} \tau \leq 0 .
\end{aligned}
$$

(C) We have

$$
\begin{aligned}
& \left|P_{0}(\tau, \xi)-i P_{1}(\tau, \xi)-P_{2}(\tau, \xi)\right| \\
& \quad \geq \operatorname{const}|\operatorname{Im} \tau|\left[(|\tau|+|\xi|)^{m-1}+(|\tau|+|\xi|)^{m-3}\right]+\left|P_{1}(\tau, \xi)\right|, \quad \operatorname{Im} \tau \leq 0,
\end{aligned}
$$

and, in addition, the polynomial $P_{1}$ has no common real roots with the polynomials $P_{0}$, $P_{2}$, and $P_{0}-P_{2}$.

Proof. $(\mathrm{A}) \rightarrow(\mathrm{B})$. The left-hand side of (5.3) equals

$$
-\operatorname{Im}\left(P_{0} \overline{P_{1}}\right)-\operatorname{Im}\left(P_{1} \overline{P_{2}}\right)+\left|P_{1}\right|^{2} .
$$

Using Proposition 2.2, we obtain the desired result.

$(\mathrm{B}) \rightarrow(\mathrm{A})$. In (5.3), let us replace $(\xi, \tau)$ with $(\rho \xi, \rho \tau)$, divide the resulting inequality by $\rho^{2 m-1}$, and pass to the limit as $\rho \rightarrow \infty$. We obtain the inequality

$$
-\operatorname{Im}\left(P_{0}(\tau, \xi) \overline{P_{1}(\tau, \xi)}\right) \geq \mathrm{const}|\operatorname{Im} \tau|(|\tau|+|\xi|)^{2 m-2}, \quad \operatorname{Im} \tau \leq 0,
$$

and Proposition 2.2 implies that $P_{0}$ and $P_{1}$ form a strictly hyperbolic pair. Similarly, dividing by $\rho^{2 m-3}$ and passing to the limit as $\rho \rightarrow 0$ we obtain the inequality

$$
-\operatorname{Im}\left(P_{1}(\tau, \xi) \overline{P_{2}(\tau, \xi)}\right) \geq \mathrm{const}|\operatorname{Im} \tau|(|\tau|+|\xi|)^{2 m-4}, \quad \operatorname{Im} \tau \leq 0,
$$

which implies that $P_{1}$ and $P_{2}$ form a strictly hyperbolic pair.

$(\mathrm{A}),(\mathrm{B}) \rightarrow(\mathrm{C})$. Since all three terms on the right-hand side of (5.3) are positive, this inequality can be rewritten as the following three inequalities:

$$
\begin{array}{rlrl}
-\operatorname{Im}\left(P_{0}(\tau, \xi)\right. & \left.-i P_{1}(\tau, \xi)-P_{2}(\tau, \xi)\right) \overline{P_{1}(\tau, \xi)} & \\
& \geq \text { const }|\operatorname{Im} \tau|(|\tau|+|\xi|)^{2 m-2}, \quad \operatorname{Im} \tau \leq 0, \\
-\operatorname{Im}\left(P_{0}(\tau, \xi)\right. & \left.-i P_{1}(\tau, \xi)-P_{2}(\tau, \xi)\right) \overline{P_{1}(\tau, \xi)} & \\
& \geq \text { const }|\operatorname{Im} \tau|(|\tau|+|\xi|)^{2 m-4}, \quad \operatorname{Im} \tau \leq 0, \\
-\operatorname{Im}\left(P_{0}(\tau, \xi)\right. & \left.-i P_{1}(\tau, \xi)-P_{2}(\tau, \xi)\right) \overline{P_{1}(\tau, \xi)} & \\
& \geq\left|P_{1}(\tau, \xi)\right|^{2}, & \operatorname{Im} \tau \leq 0 .
\end{array}
$$

The left-hand sides of the first two inequalities can be estimated from above by

$$
\text { const }(|\tau|+|\xi|)^{m-1}\left|P_{0}(\tau, \xi)-i P_{1}(\tau, \xi)-P_{2}(\tau, \xi)\right| \text {. }
$$

The left-hand side of the third inequality can be estimated from above by

$$
\left|P_{1}(\tau, \xi)\right| \cdot\left|P_{0}(\tau, \xi)-i P_{1}(\tau, \xi)-P_{2}(\tau, \xi)\right| .
$$

As a result, we come to (5.4).

Strict hyperbolicity of the pairs $P_{0}, P_{1}$ and $P_{1}, P_{2}$ implies that $P_{1}$ has no common roots with $P_{0}$, nor with $P_{2}$. Next, condition (A) implies all the assumptions of Theorem 
4.3, hence the stability of the polynomial (5.1). Since this polynomial has no real roots, the polynomials $P_{1}:=\mathcal{Q}$ and $P_{0}-P_{2}:=\mathcal{P}$ cannot have common real roots.

$(\mathrm{C}) \rightarrow(\mathrm{A})$. First we note that inequality (5.4) implies the absence of roots of the polynomial $\mathcal{R}(\tau, \xi)$ in the open lower half-plane. The absence of real roots of this polynomial immediately follows from condition (C). Hence the polynomial $\mathcal{R}(\tau, \xi)$ is stable and all three conditions in Theorem 5.1 hold. According to this theorem, all three polynomials $P_{0}, P_{1}$ and $P_{2}$ are (nonstrictly) hyperbolic and $P_{0}, P_{1}$ and $P_{1}, P_{2}$ are (nonstrictly) hyperbolic pairs. According to condition ( $\left.\mathrm{iii}_{2}\right)$, the polynomial $P_{1}$ is strictly hyperbolic. Strict hyperbolicity of $P_{0}$ and $P_{2}$ follows from (5.4). Indeed, replace $(\xi, \tau)$ with $(\rho \xi, \rho \tau)$ in (5.4) and ignore the last term on the right-hand side. If we divide the resulting inequality by $\rho^{m}$ and pass to the limit as $\rho \rightarrow \infty$, we obtain

$$
\left|P_{0}(\tau, \xi)\right| \geq \operatorname{const}|\operatorname{Im} \tau|(|\tau|+|\xi|)^{m-1}, \quad \operatorname{Im} \tau \leq 0 .
$$

Dividing by $\rho^{m-2}$ and passing to the limit as $\rho \rightarrow 0$ we obtain

$$
\left|P_{2}(\tau, \xi)\right| \geq \operatorname{const}|\operatorname{Im} \tau|(|\tau|+|\xi|)^{m-2}, \quad \operatorname{Im} \tau \leq 0 .
$$

It is well known (see, e.g., 9, Chapter I, Proposition 2.2]) that these inequalities imply strict hyperbolicity of $P_{0}$ and $P_{2}$. Since, by the assumptions, the pairs $P_{0}, P_{1}$ and $P_{1}, P_{2}$ have no common real roots, the separation of roots will actually be strict. The theorem is proved.

5.2. Analyzing condition $\left(\mathbf{I V}_{0}\right)$ for $N=3$. In this case (4.13) takes the form

$$
\Delta_{N}(\tilde{\eta}, Z)=\gamma_{1}\left[P_{0}, P_{1}\right](\tilde{\eta}) Z^{2}+\left(\gamma_{1} \gamma_{2}\left[P_{1}, P_{2}\right](\tilde{\eta})-\gamma_{3}\left[P_{0}, P_{3}\right](\tilde{\eta})\right) Z+\gamma_{2} \gamma_{3}\left[P_{2}, P_{3}\right](\tilde{\eta})
$$

and

$$
\Delta_{N}^{\prime}(\tilde{\eta}, Z)=2 \gamma_{1}\left[P_{0}, P_{1}\right](\tilde{\eta}) Z+\gamma_{1} \gamma_{2}\left[P_{1}, P_{2}\right](\tilde{\eta})-\gamma_{3}\left[P_{0}, P_{3}\right](\tilde{\eta}) .
$$

Therefore, if

$$
\gamma_{1} \gamma_{2}\left[P_{1}, P_{2}\right](\tilde{\eta})-\gamma_{3}\left[P_{0}, P_{3}\right](\tilde{\eta})>0,
$$

then the polynomial (5.5) is positive. If (5.7) does not hold, the derivative (5.6) has a nonzero root

$$
Z_{1}(\tilde{\eta})=\frac{\gamma_{3}\left[P_{0}, P_{3}\right](\tilde{\eta})-\gamma_{1} \gamma_{2}\left[P_{1}, P_{2}\right](\tilde{\eta})}{2 \gamma_{1}\left[P_{0}, P_{1}\right](\tilde{\eta})},
$$

and we have the condition

$$
-\frac{1}{4} \frac{\left(\gamma_{3}\left[P_{0}, P_{3}\right](\tilde{\eta})-\gamma_{1} \gamma_{2}\left[P_{1}, P_{2}\right](\tilde{\eta})\right)^{2}}{\gamma_{1}\left[P_{0}, P_{1}\right](\tilde{\eta})}+\gamma_{2} \gamma_{3}\left[P_{2}, P_{3}\right](\tilde{\eta})>0
$$

Therefore,

$$
\gamma_{3}\left[P_{0}, P_{3}\right](\tilde{\eta})<2 \sqrt{\gamma_{1} \gamma_{2} \gamma_{3}} \sqrt{\left[P_{1}, P_{2}\right]\left[P_{0}, P_{1}\right]}(\tilde{\eta})+\gamma_{1} \gamma_{2}\left[P_{1}, P_{2}\right](\tilde{\eta}) .
$$

Consider this expression at the point $\tilde{\eta}=(1,0)$. Since $\left[P_{i}, P_{j}\right](1,0)=j-i$, we have

$$
3 \gamma_{3}<2 \sqrt{\gamma_{1} \gamma_{2} \gamma_{3}}+\gamma_{1} \gamma_{2}
$$

or

$$
\left(\sqrt{\frac{\gamma_{1} \gamma_{2}}{\gamma_{3}}}-1\right)\left(\sqrt{\frac{\gamma_{1} \gamma_{2}}{\gamma_{3}}}+3\right)>0
$$

which implies the Routh-Hurwitz inequality (1.11). 


\section{Strictly hyperbolic POLYNOMials \\ WITH A SMALL AND A LARGE PARAMETER}

As was already mentioned in [4, 5] in the case of simplest polynomial pencils, there is a close relation between stable pencils and symbols of hyperbolic operators with small parameters that allow for a meaningful Cauchy problem. Our goal here is to study strictly hyperbolic partial differential equations with variable coefficients containing powers of a small (large) parameter. In this section we will start with studying a certain class of hyperbolic polynomials (pencils) which later will play the role of symbols of the corresponding partial differential operators.

6.1. The structure of polynomials with a small parameter. We will consider polynomials of the form

$$
R(\tau, \xi, \varepsilon)=\sum_{j=0}^{N}(-i)^{j} \varepsilon^{N-j} P^{(j)}(\tau, \xi)
$$

where $P^{(j)}$ is given in the form

$$
P^{(j)}(\tau, \xi)=\gamma_{j} P_{j}(\tau, \xi)+P^{\prime(j)}(\tau, \xi), \quad j=1, \ldots, N
$$

$P_{j}(\tau, \xi)$ is a homogeneous polynomials of degree $m-j, \gamma_{j}>0, \gamma_{0}=1$, and $P^{\prime(j)}(\tau, \xi)$ is a complex polynomial of degree at most $m-j-1$.

Later we will assume (without specially mentioning this) that

1. For $j=1, \ldots, N$ the polynomial $P_{j}(\tau, \xi)$ is resolved with respect to $\tau^{m-j}$, and $P_{j}(1,0)=1$.

2. The polynomials $P_{j}(\tau, \xi)$ have real coefficients.

The polynomial

$$
\mathcal{R}(\tau, \xi, \varepsilon):=\varepsilon^{N} P_{0}(\tau, \xi)+\sum_{j=1}^{N} \varepsilon^{N-j}(-1)^{j} \gamma_{j} P_{j}(\tau, \xi)
$$

is called the leading part of (6.1). If we assign to $(\tau, \xi)$ weight 1 and to $\varepsilon$ weight -1 , (6.2) becomes a quasihomogeneous polynomial of order $m-N$.

Depending on the parity of $N$ we will write the polynomial (5.1) and its leading part (6.2) in the following form.

For $N$ even we will write

$$
R(\tau, \xi, \varepsilon)=P(\tau, \xi, \varepsilon)-i \varepsilon Q(\tau, \xi, \varepsilon)
$$

where

$$
P(\tau, \xi, \varepsilon)=\sum_{j=0}^{\frac{N}{2}}(-1)^{j} \varepsilon^{N-2 j} P^{(2 j)}, \quad Q(\tau, \xi, \varepsilon)=\sum_{j=0}^{\frac{N-2}{2}}(-1)^{j} \varepsilon^{N-2-2 j} P^{(2 j+1)}
$$

Separating from the polynomials in (6.3) their leading parts, we write

$$
\begin{aligned}
& P(\tau, \xi, \varepsilon)=\mathcal{P}(\tau, \xi, \varepsilon)+P^{\prime}(\tau, \xi, \varepsilon), \\
& Q(\tau, \xi, \varepsilon)=\mathcal{Q}(\tau, \xi, \varepsilon)+Q^{\prime}(\tau, \xi, \varepsilon),
\end{aligned}
$$


where

$$
\begin{array}{ll}
\mathcal{P}=\sum_{j=0}^{\frac{N}{2}}(-1)^{j} \gamma_{2 j} \varepsilon^{N-2 j} P_{2 j}, & P^{\prime}=\sum_{j=0}^{\frac{N}{2}}(-1)^{j} \varepsilon^{N-2 j} P^{\prime(2 j)}, \\
\mathcal{Q}=\sum_{j=0}^{\frac{N-2}{2}}(-1)^{j} \gamma_{2 j+1} \varepsilon^{N-2-2 j} P_{2 j+1}, & Q^{\prime}=\sum_{j=0}^{\frac{N-2}{2}}(-1)^{j} \varepsilon^{N-2-2 j} P^{\prime(2 j+1)} .
\end{array}
$$

Similarly, for $N$ odd, we set

$$
R(\tau, \xi, \varepsilon):=\varepsilon P(\tau, \xi, \varepsilon)-i Q(\tau, \xi, \varepsilon)
$$

where

$$
P(\tau, \xi, \varepsilon)=\sum_{j=0}^{\frac{N-1}{2}}(-1)^{j} \varepsilon^{N-1-2 j} P^{(2 j)}, \quad Q(\tau, \xi, \varepsilon)=\sum_{j=0}^{\frac{N-1}{2}}(-1)^{j} \varepsilon^{N-1-2 j} P^{(2 j+1)} .
$$

For the leading and lowest parts in (6.5) we obtain the following expressions:

$$
\begin{array}{rlrl}
\mathcal{P} & =\sum_{j=0}^{\frac{N-1}{2}}(-1)^{j} \gamma_{2 j} \varepsilon^{N-1-2 j} P_{2 j}, & P^{\prime} & =\sum_{j=0}^{\frac{N-1}{2}}(-1)^{j} \varepsilon^{N-1-2 j} P^{\prime(2 j)}, \\
\mathcal{Q} & =\sum_{j=0}^{\frac{N-1}{2}}(-1)^{j} \gamma_{2 j+1} \varepsilon^{N-1-2 j} P_{2 j+1}, & Q^{\prime}=\sum_{j=0}^{\frac{N-1}{2}}(-1)^{j} \varepsilon^{N-1-2 j} P^{\prime(2 j+1)} .
\end{array}
$$

For $\varepsilon=1$ we retain the notation $\mathcal{R}(\tau, \xi), \mathcal{P}(\tau, \xi)$, and $\mathcal{Q}(\tau, \xi)$.

6.2. Estimates for polynomials with a small parameter. We start with a modification of Theorem 4.3 to the case of of polynomials depending on a small parameter $\varepsilon$.

Theorem 6.1. Let polynomials $P_{j}(\tau, \xi)$ satisfy conditions 1 and 2 in 6.1. Then the following conditions are equivalent:

(A) Conditions (I), $\left(\mathrm{II}_{0}\right),\left(\mathrm{III}_{0}\right)$, and $\left(\mathrm{IV}_{0}\right)$ (see Theorem 4.3) hold.

(B) Polynomials $\mathcal{P}(\sigma, \xi)$ and $\mathcal{Q}(\sigma, \xi)$ have no common nonzero real roots and

$$
\begin{aligned}
C^{-1}|\operatorname{Im} \tau|(|\tau| & +|\xi|)^{2 m-2 N}(1+\varepsilon|\tau|+\varepsilon|\xi|)^{2 N-2} \leq-\operatorname{Im}(\mathcal{P}(\tau, \xi, \varepsilon) \overline{\mathcal{Q}(\tau, \xi, \varepsilon)}) \\
& \leq C|\operatorname{Im} \tau|(|\tau|+|\xi|)^{2 m-2 N}(1+\varepsilon|\tau|+\varepsilon|\xi|)^{2 N-2}, \quad \operatorname{Im} \tau \leq 0 .
\end{aligned}
$$

Proof. According to Theorem 4.3 condition (A) is equivalent to the absence of common real roots of polynomials $\mathcal{P}$ and $\mathcal{Q}$ and inequality (4.10) (which coincides with (6.6) when $\varepsilon=1)$ :

$$
\begin{aligned}
& C^{-1}|\operatorname{Im} \tau|(|\tau|+|\xi|)^{2 m-2 N}(1+|\tau|+|\xi|)^{2 N-2} \\
& \quad \leq-\operatorname{Im}(\mathcal{P}(\tau, \xi) \overline{\mathcal{Q}(\tau, \xi)}) \leq C|\operatorname{Im} \tau|(|\tau|+|\xi|)^{2 m-2 N}(1+|\tau|+|\xi|)^{2 N-2} .
\end{aligned}
$$

In this inequality we replace $(\tau, \xi)$ with $(\varepsilon \tau, \varepsilon \xi)$. Then the left- and the right-hand side in (6.7) become, respectively, the left- and the right-hand side of (6.6) multiplied by $\varepsilon^{2 m-2 N+1}$. According to (6.3) and (6.5), for $N$ even we get

$$
\mathcal{P}(\varepsilon \tau, \varepsilon \xi)=\varepsilon^{m-N} \mathcal{P}(\tau, \xi, \varepsilon), \quad \mathcal{Q}(\varepsilon \tau, \varepsilon \xi)=\varepsilon^{m-N+1} \mathcal{Q}(\tau, \xi, \varepsilon),
$$

and for $N$ odd

$$
\mathcal{P}(\varepsilon \tau, \varepsilon \xi)=\varepsilon^{m-N+1} \mathcal{P}(\tau, \xi, \varepsilon), \quad \mathcal{Q}(\varepsilon \tau, \varepsilon \xi)=\varepsilon^{m-N} \mathcal{Q}(\tau, \xi, \varepsilon) .
$$

Therefore, the middle term in (6.7) becomes the middle term in (6.6) multiplied by $\varepsilon^{2 m-2 N+1}$. Dividing by $\varepsilon^{2 m-2 N+1}$ we obtain (6.6). 
Using the inequalities

$$
\begin{aligned}
-\operatorname{Im}(\mathcal{R}(\tau, \xi, \varepsilon) \overline{\mathcal{Q}(\tau, \xi, \varepsilon)}) & =-\operatorname{Im}(\mathcal{P}(\tau, \xi, \varepsilon) \overline{\mathcal{Q}(\tau, \xi, \varepsilon)})+\varepsilon|\mathcal{Q}(\tau, \xi, \varepsilon)|^{2}, & & N \text { even, } \\
\operatorname{Re}(\mathcal{R}(\tau, \xi, \varepsilon) \overline{\mathcal{P}(\tau, \xi, \varepsilon)}) & =-\operatorname{Im}(\mathcal{P}(\tau, \xi, \varepsilon) \overline{\mathcal{Q}(\tau, \xi, \varepsilon)})+\varepsilon|\mathcal{P}(\tau, \xi, \varepsilon)|^{2}, & & N \text { odd },
\end{aligned}
$$

we can reformulate Theorem 6.1 as follows.

Theorem 6.2. Let polynomials $P_{j}(\tau, \xi)$ satisfy conditions 1 and 2 in 6.1 . Then the following conditions are equivalent:

(A) Conditions (I), $\left(\mathrm{II}_{0}\right),\left(\mathrm{III}_{0}\right)$, and $\left(\mathrm{IV}_{0}\right)$ (see Theorem 4.3$)$ hold.

$(\tilde{\mathrm{B}})$ The polynomials $\mathcal{P}(\sigma, \xi)$ and $\mathcal{Q}(\sigma, \xi)$ have no common nonzero real roots and

$$
\begin{aligned}
& C^{-1}|\operatorname{Im} \tau|(|\tau|+|\xi|)^{2 m-2 N}(1+\varepsilon|\tau|+\varepsilon|\xi|)^{2 N-2}+\varepsilon|\mathcal{Q}(\tau, \xi, \varepsilon)|^{2} \\
& \quad \leq-\operatorname{Im}(\mathcal{R}(\tau, \xi, \varepsilon) \overline{\mathcal{Q}(\tau, \xi, \varepsilon)}) \\
& \quad \leq C|\operatorname{Im} \tau|(|\tau|+|\xi|)^{2 m-2 N}(1+\varepsilon|\tau|+\varepsilon|\xi|)^{2 N-2}+\varepsilon|\mathcal{Q}(\tau, \xi, \varepsilon)|^{2}, \quad \operatorname{Im} \tau \leq 0,
\end{aligned}
$$

for $N$ even;

$$
\begin{aligned}
& C^{-1}|\operatorname{Im} \tau|(|\tau|+|\xi|)^{2 m-2 N}(1+\varepsilon|\tau|+\varepsilon|\xi|)^{2 N-2}+\varepsilon|\mathcal{P}(\tau, \xi, \varepsilon)|^{2} \\
& \quad \leq \operatorname{Re}(\mathcal{R}(\tau, \xi, \varepsilon) \overline{\mathcal{P}(\tau, \xi, \varepsilon)}) \\
& \quad \leq C|\operatorname{Im} \tau|(|\tau|+|\xi|)^{2 m-2 N}(1+\varepsilon|\tau|+\varepsilon|\xi|)^{2 N-2}+\varepsilon|\mathcal{P}(\tau, \xi, \varepsilon)|^{2}, \quad \operatorname{Im} \tau \leq 0,
\end{aligned}
$$

for $N$ odd.

Estimates (6.8) and (6.9) can be easily generalized to the case where the polynomials (6.1), (6.4) are not homogeneous.

Theorem 6.3. For a not necessarily homogeneous polynomial $R(\tau, \xi, \varepsilon)$ of the form (6.1), (6.4) satisfying conditions 1 and 2 in 6.1, the following conditions are equivalent:

(A) The leading parts of the polynomials $\mathcal{P}$ and $\mathcal{Q}$ satisfy conditions $(\mathrm{I}),\left(\mathrm{II}_{0}\right),\left(\mathrm{III}_{0}\right)$, and $\left(\mathrm{IV}_{0}\right)$ (see Theorem 4.3).

$(\hat{\mathrm{B}})$ The polynomials $\mathcal{P}(\sigma, \xi)$ and $\mathcal{Q}(\sigma, \xi)$ have no common nonzero real roots and there exists $\gamma_{0}<0$ such that the following estimates hold:

$$
\begin{aligned}
& C_{1}^{-1}|\operatorname{Im} \tau|(|\tau|+|\xi|)^{2 m-2 N}(1+\varepsilon|\tau|+\varepsilon|\xi|)^{2 N-2}+\varepsilon|Q(\tau, \xi, \varepsilon)|^{2} \\
& \quad \leq-\operatorname{Im}(R(\tau, \xi, \varepsilon) \overline{Q(\tau, \xi, \varepsilon)}) \\
& \quad \leq C_{1}|\operatorname{Im} \tau|(|\tau|+|\xi|)^{2 m-2 N}(1+\varepsilon|\tau|+\varepsilon|\xi|)^{2 N-2}+\varepsilon|Q(\tau, \xi, \varepsilon)|^{2}, \quad \operatorname{Im} \tau \leq \gamma_{0},
\end{aligned}
$$

for $N$ even;

$$
\begin{aligned}
& C_{1}^{-1}|\operatorname{Im} \tau|(|\tau|+|\xi|)^{2 m-2 N}(1+\varepsilon|\tau|+\varepsilon|\xi|)^{2 N-2}+\varepsilon|P(\tau, \xi, \varepsilon)|^{2} \\
& \quad \leq \operatorname{Re}(R(\tau, \xi, \varepsilon) \overline{P(\tau, \xi, \varepsilon)}) \\
& \quad \leq C_{1}|\operatorname{Im} \tau|(|\tau|+|\xi|)^{2 m-2 N}(1+\varepsilon|\tau|+\varepsilon|\xi|)^{2 N-2}+\varepsilon|P(\tau, \xi, \varepsilon)|^{2}, \quad \operatorname{Im} \tau \leq \gamma_{0},
\end{aligned}
$$

for $N$ odd.

Proof. ( $\tilde{\mathrm{B}}) \rightarrow(\mathrm{A})$. Let the estimates (6.10), (6.11) hold. In these estimates, replace $(\tau, \xi, \varepsilon)$ with $\left(\rho \tau, \rho \xi, \rho^{-1} \varepsilon\right)$ and note that

$$
R\left(\rho \tau, \rho \xi, \rho^{-1} \varepsilon\right)=\rho^{m-N}\left(\mathcal{R}(\tau, \xi, \varepsilon)+O\left(\rho^{-1}\right)\right) .
$$

For $N$ even we have

$$
\begin{aligned}
& P\left(\rho \tau, \rho \xi, \rho^{-1} \varepsilon\right)=\rho^{m-N}\left(\mathcal{P}(\tau, \xi, \varepsilon)+O\left(\rho^{-1}\right)\right), \\
& Q\left(\rho \tau, \rho \xi, \rho^{-1} \varepsilon\right)=\rho^{m-N+1}\left(\mathcal{Q}(\tau, \xi, \varepsilon)+O\left(\rho^{-1}\right)\right),
\end{aligned}
$$


and for $N$ odd

$$
\begin{aligned}
& P\left(\rho \tau, \rho \xi, \rho^{-1} \varepsilon\right)=\rho^{m-N+1}\left(\mathcal{P}(\tau, \xi, \varepsilon)+O\left(\rho^{-1}\right)\right), \\
& Q\left(\rho \tau, \rho \xi, \rho^{-1} \varepsilon\right)=\rho^{m-N}\left(\mathcal{Q}(\tau, \xi, \varepsilon)+O\left(\rho^{-1}\right)\right) .
\end{aligned}
$$

Dividing these inequalities by $\rho^{2 m-2 N+1}$ and passing to the limit as $\rho \rightarrow \infty$, we obtain (6.8) (respectively, (6.9)). It remains to use Theorem 6.2.

$(\mathrm{A}) \rightarrow(\tilde{\mathrm{B}})$. According to Theorem 6.2 we have $(6.8)$ and $(6.9)$. We note that

$$
\begin{aligned}
-\operatorname{Im} & (R(\tau, \xi, \varepsilon) \overline{Q(\tau, \xi, \varepsilon)})+\operatorname{Im}(\mathcal{R}(\tau, \xi, \varepsilon) \overline{\mathcal{Q}(\tau, \xi, \varepsilon)}) \\
& =-\operatorname{Im}\left(R^{\prime}(\tau, \xi, \varepsilon) \overline{Q(\tau, \xi, \varepsilon)}\right)+\operatorname{Im}\left(\mathcal{R}(\tau, \xi, \varepsilon) \overline{Q^{\prime}(\tau, \xi, \varepsilon)}\right) .
\end{aligned}
$$

The form of the polynomials on the right-hand side of (6.12) implies that it is bounded above by

$$
\operatorname{const}(|\tau|+|\xi|)^{2 m-2 N}(1+\varepsilon|\tau|+\varepsilon|\xi|)^{2 N-2} .
$$

Similarly,

$$
\varepsilon|Q(\tau, \xi, \varepsilon)|^{2} \geq \varepsilon|\mathcal{Q}(\tau, \xi, \varepsilon)|^{2}-\operatorname{const}(|\tau|+|\xi|)^{2 m-2 N}(1+\varepsilon|\tau|+\varepsilon|\xi|)^{2 N-2} .
$$

Therefore, for large $|\operatorname{Im} \tau|$ inequality (6.8) implies (6.10). One can similarly verify that for large $|\operatorname{Im} \tau|$ inequality (6.9) implies (6.11). The theorem is proved.

\subsection{Lower estimate for the polynomial $\mathcal{R}(\tau, \xi, \varepsilon)$.}

Proposition 6.1. Let polynomials $\mathcal{R}(\tau, \xi, \varepsilon), \mathcal{P}(\tau, \xi, \varepsilon)$, and $\mathcal{Q}(\tau, \xi, \varepsilon)$ satisfy inequalities (6.8) and (6.9). Then the polynomial $\mathcal{R}(\tau, \xi, \varepsilon)$ is bounded below as follows:

$$
\text { const }|\operatorname{Im} \tau|(|\tau|+|\xi|)^{m-N-1}(1+\varepsilon(|\tau|+|\xi|))^{N} \leq|\mathcal{R}(\tau, \xi, \varepsilon)|, \quad \operatorname{Im} \tau \leq 0 .
$$

Proof. First consider the case of $N$ even. Inequality (6.8) implies that

$$
\text { const }|\operatorname{Im} \tau|(|\tau|+|\xi|)^{2 m-2 N}(1+\varepsilon(|\tau|+|\xi|))^{2 N-2} \leq|\mathcal{R}(\tau, \xi, \varepsilon)| \cdot|\mathcal{Q}(\tau, \xi, \varepsilon)| .
$$

By (6.3),

$$
|\mathcal{Q}(\tau, \xi, \varepsilon)| \leq \operatorname{const}(|\tau|+|\xi|)^{m-N+1}(1+\varepsilon(|\tau|+|\xi|))^{N-2} .
$$

Substituting this estimate on the right-hand side of (6.14), we obtain (6.13).

For $N$ odd the arguments are subtler. Inequality $(6.9)$ implies that

$$
\text { const }|\operatorname{Im} \tau|(|\tau|+|\xi|)^{2 m-2 N}(1+\varepsilon(|\tau|+|\xi|))^{2 N-2} \leq|\mathcal{R}(\tau, \xi, \varepsilon)| \cdot|\mathcal{P}(\tau, \xi, \varepsilon)| .
$$

By (6.5),

$$
|\mathcal{P}(\tau, \xi, \varepsilon)| \leq \operatorname{const}(|\tau|+|\xi|)^{m-N+1}(1+\varepsilon(|\tau|+|\xi|))^{N-1} .
$$

Substituting this estimate on the right-hand side of (6.15), we obtain

$$
\text { const }|\operatorname{Im} \tau|(|\tau|+|\xi|)^{m-N-1}(1+\varepsilon(|\tau|+|\xi|))^{N-1} \leq|\mathcal{R}(\tau, \xi, \varepsilon)| \text {. }
$$

For $\varepsilon(|\tau|+|\xi|) \gg 1$ this estimate is weaker than (6.13). To improve it, we note first that (6.9) implies additionally the inequality $\varepsilon|\mathcal{P}(\tau, \xi, \varepsilon)| \leq|\mathcal{R}(\tau, \xi, \varepsilon)|$. Therefore,

$$
\text { const }|\operatorname{Im} \tau|(|\tau|+|\xi|)^{m-N-1}(1+\varepsilon(|\tau|+|\xi|))^{N-1}+\varepsilon|\mathcal{P}(\tau, \xi, \varepsilon)| \leq|\mathcal{R}(\tau, \xi, \varepsilon)| .
$$

We prove that

$$
\begin{aligned}
\varepsilon|\mathcal{P}(\tau, \xi, \varepsilon)| \geq & \delta|\operatorname{Im} \tau|(|\tau|+|\xi|)^{m-N-1}(1+\varepsilon(|\tau|+|\xi|))^{N} \\
& -C|\operatorname{Im} \tau|(|\tau|+|\xi|)^{m-N-1}(1+\varepsilon(|\tau|+|\xi|))^{N-1} \\
& \operatorname{Im} \tau \leq 0, \quad \varepsilon(|\tau|+|\xi|)>R \gg 1 .
\end{aligned}
$$

Replacing $\varepsilon|\mathcal{P}|$ with $\theta \varepsilon|\mathcal{P}|$ for $0<\theta<1$ on the left-hand side of ([6.16), using ([6.17), and making $\theta$ sufficiently small, we obtain (6.13) for $N$ odd as well. 
To prove (6.17) we need the following result.

Lemma 6.1. Let $p(\tau)$ be a polynomial in $\tau$ of degree $m$ with all roots real and distinct. Then there exists $\kappa>0$ such that

$$
|p(\tau)|>\kappa|\operatorname{Im} \tau|(1+|\tau|)^{m-1}, \quad \operatorname{Im} \tau \leq 0,
$$

where $\kappa$ depends on $m$, the maximal absolute values of coefficients of $p(\tau)$, and of the minimal distance $\delta$ between the roots of $p(\tau)$.

The proof is based on the identity

$$
-\operatorname{Im}(p(\tau) \overline{q(\tau)})=|\operatorname{Im} \tau| \sum_{j=1}^{m} \prod_{k \neq j}\left|\tau-p_{k}\right|^{2}, \quad q(\tau)=\frac{\partial p(\tau)}{\partial \tau}, \quad \operatorname{Im} \tau<0
$$

where $p_{1}, \ldots, p_{m}$ are the roots of the polynomials $p(\tau)$.

We return to the proof of (‥17). Set

$$
\xi=|\xi| \omega, \quad \tau=|\xi| \tilde{\tau}, \quad \varepsilon \mathcal{P}(\tau, \xi, \varepsilon)=\varepsilon^{N}|\xi|^{m} \tilde{\mathcal{P}}(\tilde{\tau}, \omega, \varepsilon|\xi|),
$$

where

$$
\tilde{\mathcal{P}}(\tilde{\tau}, \omega, \varepsilon|\xi|)=P_{0}(\tilde{\tau}, \omega)-\gamma_{2}(\varepsilon|\xi|)^{-2} P_{2}(\tilde{\tau}, \omega) \pm \cdots \pm \gamma_{N-1}(\varepsilon|\xi|)^{-N+1} P_{N-1}(\tilde{\tau}, \omega)
$$

We can apply Lemma 6.1 to the polynomial (6.18). It is clear that the absolute values of the coefficients of $\tilde{\mathcal{P}}$ are bounded by a constant that does not depend on $\varepsilon|\xi|$ for $\varepsilon|\xi| \gg 1$. By the stability of the polynomial $\mathcal{R}$, the polynomial $\tilde{\mathcal{P}}$ has distinct real roots, but the stability condition does not allow us to estimate the difference between the roots. Denoting by $\tilde{p}_{j}(\omega, \varepsilon|\xi|), j=1, \ldots, m$, the roots of the polynomial ([6.18) and taking into account the results of 3.1 , we see that

$$
p_{j}(\omega, \varepsilon|\xi|)=p_{j}^{0}(\omega)+O\left((\varepsilon|\xi|)^{-2}\right), \quad j=1, \ldots, m .
$$

Since the roots $p_{j}^{0}(\omega)$ are distinct, for $\varepsilon(|\tau|+|\xi|)$ sufficiently large the distance between the roots $p_{j}(\omega, \varepsilon|\xi|)$ can be estimated in terms of the distance between $p_{j}^{0}(\omega)$, and this estimate is uniform with respect to $\varepsilon|\xi|$. This shows that we can apply Lemma 6.1 to the polynomial (6.18) obtaining

$$
\begin{aligned}
\varepsilon|\mathcal{P}(\tau, \xi, \varepsilon)|= & \varepsilon^{N}|\xi|^{m}|\tilde{\mathcal{P}}(\tilde{\tau}, \omega, \varepsilon|\xi|)| \geq \kappa \varepsilon^{N}|\xi|^{m}|\operatorname{Im} \tilde{\tau}|(1+|\tilde{\tau}|)^{m-1} \\
= & \kappa \varepsilon^{N}|\operatorname{Im} \tau|(|\tau|+|\xi|)^{m-1} \geq \kappa|\operatorname{Im} \tau|(1+\varepsilon(|\tau|+|\xi|))^{N}(|\tau|+|\xi|)^{m-N-1} \\
& -C|\operatorname{Im} \tau|(1+\varepsilon(|\tau|+|\xi|))^{N-1}(|\tau|+|\xi|)^{m-N-1} .
\end{aligned}
$$

The proposition is proved.

For - Im $\tau$ sufficiently large one can prove an analogue of the estimate (6.13) for nonhomogeneous polynomials (6.1) as well; however, we will not dwell on this.

6.4. Strictly hyperbolic polynomials with a small parameter. Proposition 6.1 suggests the following definition. A quasihomogeneous (in the sense described earlier) polynomial $\mathcal{R}(\tau, \xi, \varepsilon)$ of the form (6.2) is said to be strictly hyperbolic with a small parameter if the polynomials $\mathcal{P}(\tau, \xi, \varepsilon)$ and $\mathcal{Q}(\tau, \xi, \varepsilon)$ of the form (6.3), (6.5) are real and the estimate $(\underline{6.13})$ holds.

Using dilations $(\sigma, \xi) \rightarrow(\rho \sigma, \rho \xi)$ with $\rho \rightarrow \infty$ or $\rho \rightarrow 0$ one can show that a necessary condition for the strict hyperbolicity with a small parameter of a polynomial $\mathcal{R}$ is strict hyperbolicity of the "boundary" polynomials $P_{0}$ and $P_{N}$. Sufficient conditions for strict hyperbolicity with a small parameter is strict hyperbolicity of the pairs $P_{0}, P_{1}$ and $P_{N-1}, P_{N}$ and the condition ( $\left.\mathrm{IV}_{0}\right)$, which is difficult to verify. 
A nonhomogeneous polynomial of the form (6.1) is said to be strictly hyperbolic with a small parameter if its leading part (6.2) has this property.

Remark 6.1. It is instructive to compare the definition of strict hyperbolicity with a small parameter with the definition of ellipticity with a small parameter (see [8, 10]).

A polynomial

$$
P(\eta, \varepsilon)=\varepsilon^{N} P_{0}(\eta)+\varepsilon^{N-1} P_{1}(\eta)+\cdots+P_{N}(\eta)
$$

where $P_{0}, \ldots, P_{N}$ are homogeneous polynomials of order $m, \ldots, m-N$, is said to be elliptic with a small parameter if

$$
|P(\eta, \varepsilon)|>\operatorname{const}|\eta|^{m-N}(1+\varepsilon|\eta|)^{N} .
$$

As noted in [10, the polynomial (6.19) is elliptic with a small parameter if and only if the polynomials $P_{0}$ and $P_{N}$ are elliptic and the polynomial $P(\omega, \varepsilon),|\omega|=1$, does not take the zero value for $\varepsilon>0$.

In the case of variable symbols $P(y ; \eta, \varepsilon)$ the condition of ellipticity with a small parameter for each $y \in \mathbb{R}^{n+1}$ allows one to obtain results in the case of variable coefficients.

As we will see later, contrary to the case of elliptic equations, in the case of hyperbolic equations one cannot consider just the symbols $R(y ; \eta, \varepsilon)$ satisfying, for each $y \in \mathbb{R}^{n+1}$, the condition of hyperbolicity with a small parameter. The possibility to pass to variable coefficients is guaranteed by additional assumptions about strict hyperbolicity of pairs $P_{0}, P_{1}$ and $P_{N-1}, P_{N}$, and condition (IV).

Therefore, in the elliptic case the passage to variable coefficients is significantly simpler than in the hyperbolic case.

6.5. Strictly hyperbolic polynomials with a large parameter. To each polynomial $R(\tau, \xi, \varepsilon)$ with a small parameter $\varepsilon \rightarrow 0$ one can associate a polynomial $\tilde{R}(\tau, \xi, \lambda)$ with a large parameter $\lambda \rightarrow+\infty$ :

$$
\tilde{R}(\tau, \xi, \lambda)=\lambda^{N} R\left(\tau, \xi, \frac{1}{\lambda}\right)=P_{0}(\tau, \xi)+\sum_{j=1}^{N}(-i)^{j} \lambda^{j} P^{(j)}(\tau, \xi) .
$$

The leading part of the polynomial (6.20) is defined naturally:

$$
\tilde{\mathcal{R}}(\tau, \xi, \lambda):=P_{0}(\tau, \xi)+\sum_{j=1}^{N}(-i)^{j} \gamma_{j} \lambda^{j} P_{j}(\tau, \xi) .
$$

The polynomial (6.21) is said to be strictly hyperbolic with a large parameter if the following estimate holds:

$$
|\tilde{\mathcal{R}}(\tau, \xi, \lambda)| \geq \text { const }|\operatorname{Im} \tau|(|\tau|+|\xi|)^{m-N-1}(\lambda+|\tau|+|\xi|)^{N} .
$$

It is natural to write the polynomials (6.20) and (6.21) in the following form:

$$
\tilde{R}(\tau, \xi, \lambda)=\tilde{P}(\tau, \xi, \lambda)-i \lambda \tilde{Q}(\tau, \xi, \lambda) .
$$

Polynomials in (6.22) are related to the the polynomials in (6.3) and (6.5) as follows:

$$
\begin{aligned}
& \tilde{\mathcal{P}}(\tau, \xi, \lambda)=\lambda^{N} \mathcal{P}\left(\tau, \xi, \frac{1}{\lambda}\right), \quad \tilde{\mathcal{Q}}(\tau, \xi, \lambda)=\lambda^{N-1} \mathcal{Q}\left(\tau, \xi, \frac{1}{\lambda}\right), \quad N \text { even }, \\
& \tilde{\mathcal{P}}(\tau, \xi, \lambda)=\lambda^{N-1} \mathcal{P}\left(\tau, \xi, \frac{1}{\lambda}\right), \quad \tilde{\mathcal{Q}}(\tau, \xi, \lambda)=\lambda^{N} \mathcal{Q}\left(\tau, \xi, \frac{1}{\lambda}\right), \quad N \text { odd } .
\end{aligned}
$$

Using definitions (6.20), (6.21) and relations (6.22), (6.23), one can extend all results of this section about polynomials with a small parameter to the case of polynomials with a large parameter. We leave this to the reader as a simple exercise. 


\section{The Cauchy problem for a Hyperbolic EQUATION WITH A SMALL PARAMETER AT HIGHEST DERIVATIVES}

In this section we discuss applications of the previous results to the study of (homogeneous) Cauchy problem for real equations with constant or variable coefficients and with a small or large parameter. Since the two cases are similar, we will concentrate on equations with a small parameter.

7.1. Statement of the problem. Notation. Consider the equation

$$
R(y, D, \varepsilon) u(y)=f(y) .
$$

Here $y=(t, x) \in \mathbb{R}^{n+1}, D=\left(D_{t}, D_{x}\right)=\left(D_{t}, D_{1}, \ldots, D_{n}\right)$ and, as usual, $D_{t}=\frac{1}{i} \frac{\partial}{\partial t}$, $D_{k}=\frac{1}{i} \frac{\partial}{\partial x_{k}}$.

We denote by $R(y, \tau, \xi, \varepsilon)$ the symbol of the operator (7.1) and assume that it has the structure described in 6.1 , so that

$$
R(y, \tau, \xi, \varepsilon)=\sum_{j=0}^{N}(-i)^{j} \varepsilon^{N-j} P^{(j)}(y, \tau, \xi) .
$$

The symbols $P^{(j)}(y, \tau, \xi), j=1, \ldots, N$, are polynomials in $(\tau, \xi)$ of degree $m-j$ with coefficients smoothly depending on variables $y:=(t, x)$. We assume that the coefficients of the polynomials (8.2) do not depend on $y$ for $|y|$ large.

We will write the leading homogeneous part $P^{(j)}$ in the form $\gamma_{j} P_{j}(y, \tau, \xi)$, where the symbol $P_{j}(y, \tau, \xi)$ is homogeneous of degree $m-j$ in $(\tau, \xi)$, and $\gamma_{j}$ is a positive constant, $\gamma_{0}=1$.

Furthermore, we will always assume that

1. For $j=1, \ldots, N$ the symbol $P_{j}(y, \tau, \xi)$ is resolved with respect to $\tau^{m-j}$, and $P_{j}(y, 1,0) \equiv 1$.

2. The symbols $P_{j}(y, \tau, \xi)$ have real coefficients.

Remark 7.1. The condition that the symbols $\gamma_{j} P_{j}(y, 1,0)$ do not depend on $y$ can be weakened by replacing, for example, the constants $\gamma_{j}$ with functions $\gamma_{j}(y)$ satisfying, for each fixed $y$, the Routh-Hurwitz conditions. We will not dwell on this.

The symbol

$$
\mathcal{R}(y, \tau, \xi, \varepsilon):=\varepsilon^{N} P_{0}(y, \tau, \xi)+\sum_{j=1}^{N} \varepsilon^{N-j}(-i)^{j} \gamma_{j} P_{j}(y, \tau, \xi)
$$

is called the leading part of the symbol (7.2).

Following 6.1, we will write the operator (7.1) and its leading symbol (7.3) in the following form (depending on the parity of $N$ ):

$$
\begin{array}{rlrl}
R(y, D, \varepsilon) & =P(y, D, \varepsilon)-i \varepsilon Q(y, D, \varepsilon), & & N \text { even, } \\
R(y, D, \varepsilon) & =\varepsilon P(y, D, \varepsilon)-i Q(y, D, \varepsilon), & N \text { odd, } \\
\mathcal{R}(y, \tau, \xi, \varepsilon) & =\mathcal{P}(y, \tau, \xi, \varepsilon)-i \varepsilon \mathcal{Q}(y, \tau, \xi, \varepsilon), & & N \text { even, } \\
\mathcal{R}(y, \tau, \xi, \varepsilon) & =\varepsilon \mathcal{P}(y, \tau, \xi, \varepsilon)-i \mathcal{Q}(y, \tau, \xi, \varepsilon), & & N \text { odd. }
\end{array}
$$

The explicit form of the symbols $\mathcal{P}(y, \tau, \xi, \varepsilon)$ and $\mathcal{Q}(y, \tau, \xi, \varepsilon)$ is the same as that of the polynomials $\mathcal{P}(\tau, \xi, \varepsilon)$ and $\mathcal{Q}(\tau, \xi, \varepsilon)$ in 6.1 , and we will not repeat the corresponding formulas.

We will use the standard function spaces. Denote by $H^{s}:=H^{s}\left(\mathbb{R}^{n+1}\right)$ the usual Sobolev space with the norm $\|\cdot\|_{s}$, and by $H_{[\gamma]}^{s}$ the set of all functions $u$ such that $e^{\gamma t} u \in H^{s}$. As usual, for $s=0$ we will omit the corresponding index. 
For the norm in $H_{[\gamma]}^{s}$ one can take

$$
\left(\int|\hat{u}(\sigma+i \gamma, \xi)|^{2}\left(1+|\sigma+i \gamma|^{2}+|\xi|^{2}\right)^{s} d \xi d \sigma\right)^{1 / 2}
$$

where by $\hat{u}(\sigma+i \gamma, \xi)$ we denote the (complex) Fourier transform of the function $u(t, x)$ :

$$
\hat{u}(\sigma+i \gamma, \xi)=(2 \pi)^{-n / 2} \int \exp \{i t(\sigma+i \gamma)+i(x, \xi)\} u(t, x) d t d x .
$$

We find it more convenient to use an equivalent norm defined by using pseudodifferential operators (for details, see [9] Chapter 2, Section 1.6]):

$$
\|u\|_{s[\gamma]}:=\left\|\exp (\gamma t) \delta_{s}^{+}\left(D_{t}, D_{x}\right) u\right\|
$$

where $\delta_{s}^{+}\left(D_{t}, D_{x}\right)$ is the pseudodifferential operator with the symbol

$$
\delta_{s}^{+}(\tau, \xi)=\left(i \tau+\sqrt{1+|\xi|^{2}}\right)^{s}, \quad \gamma \leq 0 .
$$

In the space $H_{[\gamma]}^{s}$ we introduce the natural inner product

$$
(u, v)_{s[\gamma]}=\left(\delta_{s}^{+}\left(D_{t}, D_{x}\right) u, \delta_{s}^{+}\left(D_{t}, D_{x}\right) v\right)_{[\gamma]},
$$

where

$$
(u, v)_{[\gamma]}=\int e^{2 \gamma t} u(y) \overline{v(y)} d y .
$$

Denote by $H_{[\gamma]+}^{s}$ the subspace of $H_{[\gamma]}^{s}$ consisting of all functions with support in the half-space $\mathbb{R}_{+}^{n+1}:=\left\{(t, x) \in \mathbb{R}^{n+1}, t \geq 0\right\}$. The detailed theory of these spaces can be found in $[9]$. Later we will use the fact that a function (distribution) $u$ belongs to $H_{[\gamma]+}^{s}$ if and only if $\delta_{s}^{+}\left(D_{t}, D_{x}\right) u \in H_{[\gamma]+}$.

Consider equation (7.1) for the right-hand side $f(y) \in H_{[\gamma]+}^{s}$. The problem of finding $u(y) \in H_{[\gamma]+}^{s+\kappa}$ with some $\kappa>0$ is called the homogeneous Cauchy problem. It is known (see, e.g., [9]) that if the symbol $P_{0}(t, x, \tau, \xi)$ is strictly hyperbolic, then the homogeneous Cauchy problem for (17.1) (with real $\varepsilon \neq 0$ ) is uniquely solvable for $\gamma \leq \gamma_{s}$ and has a solution $u(y) \in H_{[\gamma]+}^{s+m-1}$.

The main nontrivial problem arising in the study of (7.1) is to find conditions that must be imposed on the symbol (7.2) to ensure the existence of an estimate for (7.1), uniform with respect to $\varepsilon$, in terms of the right-hand side. For $N=1,2$ the corresponding estimates were obtained in 44 and 5] respectively. Using the strict hyperbolicity of $P_{2}$, the authors [5] were able to construct the formal asymptotic solution (FAS) of the homogeneous Cauchy problem. Uniform estimates for the solution of the Cauchy problem allows one to show that the formal asymptotic expression obtained using FAS is the genuine asymptotic formula for the solution. The existence of a uniform estimate makes it possible to automatically extend this result to the case of an arbitrary $N$.

In the case of the nonhomogeneous Cauchy problem the construction of FAS becomes more complicated due to the existence of boundary layers. However, the corresponding construction does not differ from the Vishik-Lyusternik constructions (see [8] or [10]), and we will not dwell on this.

To deduce estimates for (7.1) uniform in $\varepsilon$ it is natural to use the norms depending on $\varepsilon$,

$$
\|u\|_{r, s[\gamma]}:=\left\|\delta_{r}^{+}\left(D_{t}, D_{x}\right) \delta_{s}^{+}\left(\varepsilon D_{t}, \varepsilon D_{x}\right) u\right\|_{[\gamma]} .
$$

We note the obvious inequality

$$
\varepsilon^{k}\|u\|_{r, s[\gamma]} \leq\|u\|_{r-k, s+k[\gamma]},
$$

which will be used later. 
7.2. The main estimate. The case of constant coefficients. In the case of constant coefficients the required uniform estimates are obtained using the Fourier transform from Theorems 6.1 and 6.2 and Proposition 6.1

It is convenient to modify the norms $\|u\|_{s[\gamma]}$ and $\|u\|_{r, s[\gamma]}$ replacing the first with the "homogeneous" norm

$$
\|u\|_{s[\gamma]}^{\prime}:=\left\|\left(i D_{t}+\left|D_{x}\right|\right)^{s} u\right\|_{[\gamma]}=\left(\int|\hat{u}(\sigma+i \gamma, \xi)|^{2}(|i(\sigma+i \gamma)|+|\xi|)^{2 s} d \xi d \sigma\right)^{1 / 2}
$$

and the second with the norm

$$
\|u\|_{r, s[\gamma]}^{\prime}:=\left\|\delta_{s}^{+}\left(\varepsilon D_{t}, \varepsilon D_{x}\right) u\right\|_{r[\gamma]}^{\prime} .
$$

Similarly, we replace the inner product $(u, v)_{s[\gamma]}$ with

$$
(u, v)_{s[\gamma]}^{\prime}=\left(\left(i D_{t}+\left|D_{x}\right|\right)^{s} u,\left(i D_{t}+\left|D_{x}\right|\right)^{s} v\right)_{[\gamma]} .
$$

A quasihomogeneous differential operator $\mathcal{R}\left(D_{t}, D_{x}, \varepsilon\right)$ with constant coefficients is said to be strictly hyperbolic with a small parameter if its $\operatorname{symbol} \mathcal{R}(\tau, \xi, \varepsilon)$ has this property.

One can easily see that the strict hyperbolicity of the operator $\mathcal{R}\left(D_{t}, D_{x}, \varepsilon\right)$ is equivalent to the following lower bound:

$$
C^{-1}|\gamma| \cdot\|u\|_{r+m-N-1, s+N[\gamma]}^{\prime} \leq\left\|\mathcal{R}\left(D_{t}, D_{x}, \varepsilon\right) u\right\|_{r, s[\gamma]}^{\prime}
$$

Theorems 6.1, 6.2 and Proposition 6.1 can be reformulated as follows.

Proposition 7.1. Let polynomials $P_{j}(\tau, \xi)$ satisfy conditions 1,2 in 6.1 . Then the following conditions are equivalent.

(A) Conditions $(\mathrm{I}),\left(\mathrm{II}_{0}\right),\left(\mathrm{III}_{0}\right)$ and $\left(\mathrm{IV}_{0}\right)$ are satisfied (see Theorem 4.3).

(B) The polynomials $\mathcal{P}(\sigma, \xi)$ and $\mathcal{Q}(\sigma, \xi)$ have no common nonzero real roots and

$$
\begin{aligned}
C^{-1}|\gamma| \cdot\|u\|_{s+m-N, N-1,[\gamma]}^{2} & \leq-\operatorname{Im}\left(\mathcal{P}\left(D_{t}, D_{x}, \varepsilon\right) u, \mathcal{Q}\left(D_{t}, D_{x}, \varepsilon\right) u\right)_{s[\gamma]}^{\prime} \\
& \leq C|\gamma| \cdot\|u\|_{s+m-N, N-1,[\gamma]}^{2}, \quad \gamma \leq 0 .
\end{aligned}
$$

Proposition 7.2. Let polynomials $P_{j}(\tau, \xi)$ satisfy conditions 1,2 in 6.1 . Then the following conditions are equivalent.

(A) Conditions $(\mathrm{I}),\left(\mathrm{II}_{0}\right),\left(\mathrm{II}_{0}\right)$ and $\left(\mathrm{IV}_{0}\right)$ are satisfied (see Theorem 4.3).

$(\tilde{\mathrm{B}})$ The polynomials $\mathcal{P}(\sigma, \xi)$ and $\mathcal{Q}(\sigma, \xi)$ have no common nonzero real roots and the following estimates hold.

For $N$ even:

$$
\begin{aligned}
C^{-1}|\gamma| & \cdot\|u\|_{s+m-N, N-1,[\gamma]}^{2}+\varepsilon\left\|\mathcal{Q}\left(D_{t}, D_{x}, \varepsilon\right) u\right\|_{s,[\gamma]}^{2} \\
& \leq-\operatorname{Im}\left(\mathcal{R}\left(D_{t}, D_{x}, \varepsilon\right) u, \mathcal{Q}\left(D_{t}, D_{x}, \varepsilon\right) u\right)_{s[\gamma]}^{\prime} \\
& \leq C|\gamma| \cdot\|u\|_{s+m-N, N-1,[\gamma]}^{2}+\varepsilon\left\|\mathcal{Q}\left(D_{t}, D_{x}, \varepsilon\right) u\right\|_{s,[\gamma]}^{\prime 2}, \quad \gamma \leq 0
\end{aligned}
$$

for $N$ odd:

$$
\begin{aligned}
C^{-1}|\gamma| & \cdot\|u\|_{s+m-N, N-1,[\gamma]}^{2}+\varepsilon\left\|\mathcal{P}\left(D_{t}, D_{x}, \varepsilon\right) u\right\|_{s,[\gamma]}^{2} \\
& \leq \operatorname{Re}\left(\mathcal{R}\left(D_{t}, D_{x}, \varepsilon\right) u, \mathcal{P}\left(D_{t}, D_{x}, \varepsilon\right) u\right)_{s[\gamma]}^{\prime} \\
& \leq C|\gamma| \cdot\|u\|_{s+m-N, N-1,[\gamma]}^{2}+\varepsilon\left\|\mathcal{P}\left(D_{t}, D_{x}, \varepsilon\right) u\right\|_{s,[\gamma]}^{2}, \quad \gamma \leq 0 .
\end{aligned}
$$


Proposition 7.3. Under the assumptions of Proposition 6.1, we have the following lower bound:

$$
C|\gamma| \cdot\|u\|_{s+m-N-1, N,[\gamma]}^{\prime} \leq\left\|\mathcal{R}\left(D_{t}, D_{x}, \varepsilon\right) u\right\|_{s,[\gamma]}^{\prime}, \quad \gamma \leq 0 .
$$

7.3. Hyperbolic equations with a small parameter. The case of variable coefficients. Now we formulate the main estimates for equation (7.1) in the case of variable coefficients. We will assume that the operator in (7.1) is strictly hyperbolic with a small parameter. The latter condition means that at any point $y^{0} \in \mathbb{R}^{n+1}$ the leading symbol $\mathcal{R}\left(y^{0}, \tau, \xi, \varepsilon\right)$ is a strictly hyperbolic polynomial with a small parameter. As we have already mentioned earlier, this condition is too weak to obtain estimates for equations with variable coefficients. Sufficient (and equivalent) conditions for the existence of estimates are the assumptions of Theorem 4.3. Under these conditions we can consider quadratic forms that allow for the "freezing" of the coefficients. Two-sided estimates for the forms yield "estimates with the loss of smoothness" for the original equation (7.1). A similar approach for strictly hyperbolic equations was suggested in the classical papers by Leray (see the detailed exposition in [9]); applications of this approach to more general equations was developed in [11.

Theorem 7.1. Let the polynomials $P_{j}\left(y^{0}, \tau, \xi\right)$ satisfy conditions 1 and 2 in 7.1 at each point $y^{0} \in \mathbb{R}^{n+1}$. Then the following conditions are equivalent.

(A) The polynomials $\mathcal{R}\left(y^{0}, \tau, \xi, \varepsilon\right), \mathcal{P}\left(y^{0}, \tau, \xi, \varepsilon\right)$, and $\mathcal{Q}\left(y^{0}, \tau, \xi, \varepsilon\right)$ satisfy conditions (I), $\left(\mathrm{II}_{0}\right),\left(\mathrm{III}_{0}\right)$ and $\left(\mathrm{IV}_{0}\right)$ at any point $y^{0} \in \mathbb{R}^{n+1}$ (see Theorem 4.3).

$(\tilde{\mathrm{B}})$ The polynomials $\mathcal{P}\left(y^{0}, \sigma, \xi\right)$ and $\mathcal{Q}\left(y^{0} \sigma, \xi\right)$ have no common nonzero real roots, and for each $S \geq 0$ there exists $\gamma_{0}=\gamma_{0}(S)$ such that for $|s| \leq S$ the following estimates hold.

For $N$ even:

$$
\begin{aligned}
& C^{-1}|\gamma| \cdot\|u\|_{s+m-N, N-1,[\gamma]}^{2}+\varepsilon\left\|Q\left(y, D_{t}, D_{x}, \varepsilon\right) u\right\|_{s,[\gamma]}^{2} \\
& \quad \leq-\operatorname{Im}\left(R\left(y, D_{t}, D_{x}, \varepsilon\right) u, Q\left(y, D_{t}, D_{x}, \varepsilon\right) u\right)_{s[\gamma]} \\
& \quad \leq C|\gamma| \cdot\|u\|_{s+m-N, N-1,[\gamma]}^{2}+\varepsilon\left\|Q\left(y, D_{t}, D_{x}, \varepsilon\right) u\right\|_{s,[\gamma]}^{2}, \quad \gamma \leq \gamma_{0} ;
\end{aligned}
$$

for $N$ odd:

$$
\begin{aligned}
& C^{-1}|\gamma| \cdot\|u\|_{s+m-N, N-1,[\gamma]}^{2}+\varepsilon\left\|P\left(y, D_{t}, D_{x}, \varepsilon\right) u\right\|_{s,[\gamma]}^{2} \\
& \quad \leq \operatorname{Re}\left(R\left(y, D_{t}, D_{x}, \varepsilon\right) u, P\left(y, D_{t}, D_{x}, \varepsilon\right) u\right)_{s[\gamma]} \\
& \quad \leq C|\gamma| \cdot\|u\|_{s+m-N, N-1,[\gamma]}^{2}+\varepsilon\left\|P\left(y, D_{t}, D_{x}, \varepsilon\right) u\right\|_{s,[\gamma]}^{2}, \quad \gamma \leq \gamma_{0} .
\end{aligned}
$$

The main step in proving Theorem 7.1 is to estimate the quadratic form

$$
-\operatorname{Im}\left(\mathcal{P}\left(y, D_{t}, D_{x}, \varepsilon\right) u, \mathcal{Q}\left(y, D_{t}, D_{x}, \varepsilon\right) u\right)_{s[\gamma]}
$$

from above and from below in terms of similar forms with coefficients frozen at an arbitrary point $y=y^{0}$.

We will prove the following result.

Theorem 7.2. Let polynomials $P_{j}\left(y^{0}, \tau, \xi\right)$ satisfy conditions 1 and 2 in 7.1 at a point $y^{0} \in \mathbb{R}^{n+1}$. Then the following conditions are equivalent.

(A) The polynomials $\mathcal{R}\left(y^{0}, \tau, \xi, \varepsilon\right), \mathcal{P}\left(y^{0}, \tau, \xi, \varepsilon\right)$, and $\mathcal{Q}\left(y^{0}, \tau, \xi, \varepsilon\right)$ satisfy conditions $(\mathrm{I}),\left(\mathrm{II}_{0}\right),\left(\mathrm{III}_{0}\right)$ and $\left(\mathrm{IV}_{0}\right)$ at an arbitrary point $y^{0} \in \mathbb{R}^{n+1}$ (see Theorem 4.3). 
L. R. VOLEVICH AND E. V. RADKEVICH

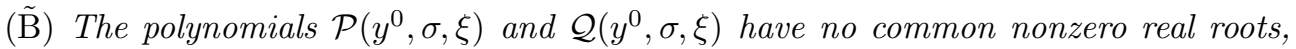
and for any $S \geq 0$ there exists $\gamma_{0}=\gamma_{0}(S)$ such that for $|s| \leq S$ we have

$$
\begin{aligned}
C^{-1}|\gamma| \cdot\|u\|_{s+m-N, N-1,[\gamma]}^{2} & \leq-\operatorname{Im}\left(\mathcal{P}\left(y, D_{t}, D_{x}, \varepsilon\right) u, \mathcal{Q}\left(y, D_{t}, D_{x}, \varepsilon\right) u\right)_{s[\gamma]} \\
& \leq C|\gamma| \cdot\|u\|_{s+m-N, N-1,[\gamma]}^{2}, \quad \gamma \leq \gamma_{0} .
\end{aligned}
$$

7.4. Uniform estimate of solutions of (7.1). As a direct consequence of Theorem 7.1 we obtain the following result.

Proposition 7.4. Under the assumptions of Theorem 7.1 we have the following estimates.

For $N$ even:

$$
|\gamma| \cdot\|u\|_{s+m-N, N-1,[\gamma]} \leq \mathrm{const}\left\|R\left(y, D_{t}, D_{x}, \varepsilon\right) u\right\|_{s+1,-1,[\gamma]} ;
$$

for $N$ odd:

$$
|\gamma| \cdot\|u\|_{s+m-N, N-1,[\gamma]} \leq \mathrm{const}\left\|R\left(y, D_{t}, D_{x}, \varepsilon\right) u\right\|_{s+1,[\gamma]} .
$$

Proof (cf. Proposition 6.1). We start with the case of $N$ even. First we estimate the form in (7.11) from above:

$$
\begin{aligned}
-\operatorname{Im}(R u, Q u)_{s[\gamma]} & =-\operatorname{Im}\left(\delta_{1}^{+}(D) \delta_{-1}^{+}(\varepsilon D) R u, \delta_{-1}^{+}(D) \delta_{1}^{+}(\varepsilon D) Q u\right)_{s[\gamma]} \\
& \leq\|R u\|_{s+1,-1[\gamma]}\|Q u\|_{s-1,1[\gamma]} .
\end{aligned}
$$

Taking into account the definition of the operator $Q$ for $N$ even and inequality (7.7), we obtain

$$
\begin{aligned}
\|Q u\|_{s-1,1[\gamma]} & \leq \sum_{j \geq 0} \varepsilon^{N-2-2 j}\left\|P^{(2 j+1)} u\right\|_{s-1,1[\gamma]} \leq \mathrm{const} \sum_{j \geq 0} \varepsilon^{N-2-2 j}\|u\|_{s+m-2-2 j, 1[\gamma]} \\
& \leq \mathrm{const} \sum_{j \geq 0}\|u\|_{s+m-N, N-1-2 j[\gamma]} \leq \mathrm{const}\|u\|_{s+m-N, N-1[\gamma]} .
\end{aligned}
$$

Substituting these estimates on the right-hand side of the inequality

$$
|\gamma| \cdot\|u\|_{s+m-N, N-1,[\gamma]}^{2} \leq-\operatorname{Im}(R u, Q u)_{s[\gamma]}
$$

we arrive at (7.14).

For $N$ odd we use the estimate

$$
\operatorname{Re}(R u, P u)_{s[\gamma]} \leq\|R u\|_{s+1,[\gamma]}\|P u\|_{s-1[\gamma]} .
$$

Taking into account the definition of the operator $P$ for $N$ odd and inequality (7.7), we obtain

$$
\begin{aligned}
\|P u\|_{s-1,[\gamma]} & \leq \sum_{j \geq 0} \varepsilon^{N-1-2 j}\left\|P^{2 j} u\right\|_{s-1,[\gamma]} \\
& \leq \text { const } \sum_{j \geq 0} \varepsilon^{N-1-2 j}\|u\|_{s+m-1-2 j,[\gamma]} \leq \text { const }\|u\|_{s+m-N, N-1,[\gamma]} .
\end{aligned}
$$

Substituting these estimates on the right-hand side of the inequality

$$
|\gamma| \cdot\|u\|_{s+m-N, N-1,[\gamma]}^{2} \leq \operatorname{Re}(R u, P u)_{s[\gamma]},
$$

we arrive at (7.15).

Remark 7.2. Inequality (17.15) is weaker than (7.14). One could try to make it stronger using an additional inequality (see the proof of Proposition 6.1)

$$
\varepsilon\|P u\|_{s,[\gamma]} \leq\|R u\|_{s,[\gamma]} .
$$

We will not dwell on this. 


\section{The PRoOf of ENERGy ESTIMATES}

8.1. Reduction of Theorem 7.1 to Theorem $\mathbf{7 . 2}$. First we note that Theorem 7.2 includes the form (7.13) containing the leading homogeneous parts $\mathcal{P}$ and $\mathcal{Q}$ of nonhomogeneous operators $P$ and $Q$. In deducing Theorem 7.1 from Theorem 7.2 we will need an analog of (7.13) for nonhomogeneous operators $P$ and $Q$. To obtain it, consider the difference of the corresponding forms setting

$$
\begin{aligned}
H_{s, \gamma}[u, u]:= & -\operatorname{Im}\left(P\left(y, D_{t}, D_{x}, \varepsilon\right) u, Q\left(y, D_{t}, D_{x}, \varepsilon\right) u\right)_{s[\gamma]} \\
& +\operatorname{Im}\left(\mathcal{P}\left(y, D_{t}, D_{x}, \varepsilon\right) u, \mathcal{Q}\left(y, D_{t}, D_{x}, \varepsilon\right) u\right)_{s[\gamma]} .
\end{aligned}
$$

We show that

$$
\left|H_{s, \gamma}[u, u]\right| \leq K\|u\|_{s+m-N, N-1,[\gamma]}^{2},
$$

where $K$ is a constant that does not depend on $\gamma$. It follows from this estimate that for a sufficiently large $-\gamma$ the inequality

$$
|\gamma| \cdot\|u\|_{s+m-N, N-1,[\gamma]}^{2} \leq-C_{1} \operatorname{Im}\left(P\left(y, D_{t}, D_{x}, \varepsilon\right) u, Q\left(y, D_{t}, D_{x}, \varepsilon\right) u\right)_{s[\gamma]}
$$

implies the inequality

$$
|\gamma| \cdot\|u\|_{s+m-N, N-1,[\gamma]}^{2} \leq-C_{2} \operatorname{Im}\left(\mathcal{P}\left(y, D_{t}, D_{x}, \varepsilon\right) u, \mathcal{Q}\left(y, D_{t}, D_{x}, \varepsilon\right) u\right)_{s[\gamma]},
$$

and vice versa. Before verifying (8.2), let us complete the deduction of Theorem 7.1 from Theorem 7.2

$(\mathrm{A}) \rightarrow(\tilde{\mathrm{B}})$. According to Theorem 7.2, condition (A) implies (8.4), which, in turn, implies (8.3). Using obvious equalities

$$
\begin{aligned}
-\operatorname{Im}(R u, Q u)_{s[\gamma]} & =-\operatorname{Im}(P u, Q u)_{s[\gamma]}+\varepsilon\|Q u\|_{s[\gamma]}^{2}, & & N \text { odd, } \\
\operatorname{Re}(R u, P u)_{s[\gamma]} & =-\operatorname{Im}(P u, Q u)_{s[\gamma]}+\varepsilon\|P u\|_{s[\gamma]}^{2}, & & N \text { even, }
\end{aligned}
$$

we obtain (7.11) and 7.12).

$(\tilde{\mathrm{B}}) \rightarrow(\mathrm{A})$. Inequalities (7.11) and (7.12) imply (8.3), which, in turn, implies (8.4). We use Theorem 7.2 to prove (A).

To prove (8.2) we write the expression (8.1) explicitly. We have

$$
\begin{aligned}
H_{s, \gamma}[u, u]= & -\operatorname{Im}\left(P^{\prime}\left(y, D_{t}, D_{x}, \varepsilon\right) u, Q\left(y, D_{t}, D_{x}, \varepsilon\right) u\right)_{s[\gamma]} \\
& +\operatorname{Im}\left(\mathcal{P}\left(y, D_{t}, D_{x}, \varepsilon\right) u, Q^{\prime}\left(y, D_{t}, D_{x}, \varepsilon\right) u\right)_{s[\gamma]} \\
:= & H_{s, \gamma}^{\prime}[u, u]+H_{s, \gamma}^{\prime \prime}[u, u],
\end{aligned}
$$

where

$$
\begin{aligned}
& H_{s, \gamma}^{\prime}[u, u]=\sum_{j, k \geq 0}(-1)^{j+k+1} \varepsilon^{2 N-2 j-2 k-2} \operatorname{Im}\left(P^{\prime(2 j)} u, Q^{(2 k+1)} u\right)_{s[\gamma]}, \\
& H_{s, \gamma}^{\prime \prime}[u, u]=\sum_{j, k \geq 0}(-1)^{j+k+1} \gamma_{2 j} \varepsilon^{2 N-2 j-2 k-2} \operatorname{Im}\left(P_{2 j} u, Q^{\prime(2 k+1)} u\right)_{s[\gamma]} .
\end{aligned}
$$

We denote by $G_{r}, G_{r}^{\prime}, G_{r}^{\prime \prime}$ various differential operators of order at most $r$ of the form

$$
G_{r}=\sum_{l+|\alpha| \leq r} \gamma^{l} h_{l \alpha}(y) D^{\alpha} .
$$

Then (8.5) is a linear combination of forms of the following type:

$$
\varepsilon^{2 N-2 j-2 k-2}\left(G_{m-2 j-1} u, G_{m-2 k-1} u\right)_{s[\gamma]} .
$$


L. R. VOLEVICH AND E. V. RADKEVICH

We integrate these forms by parts. Suppose, for example, that $j<k$. Moving $k-j$ derivatives to the right operator (for details see [9, Chapter II, Proposition 4.4.1]), we obtain an expression of the form

$$
\varepsilon^{2 N-2 j-2 k-2}\left(G_{m-j-k-1}^{\prime} u, G_{m-j-k-1}^{\prime \prime} u\right)_{s[\gamma]} .
$$

The absolute values of these forms can easily be estimated in terms of the right-hand side of (8.2)).

Similarly, (8.6) is a linear combination of forms of the following type:

$$
\varepsilon^{2 N-2 j-2 k-2}\left(H_{m-2 j} u, H_{m-2 k-2} u\right)_{s[\gamma]} .
$$

Integrating these forms by parts moving $k-j+1$ derivatives to the right operator, we again obtain an expression of the form (8.7).

8.2. The proof of Theorem 7.2 Necessity of condition (A) for the estimate (17.13) to hold. The main step in the proof is the following statement (about strictly hyperbolic equations see [9, Chapter II, Proposition 4.2]).

Lemma 8.1. Let the first inequality in (7.13) hold for $s=0$ and $\gamma \leq \gamma_{0}$ with a constant $C$ independent of $\gamma$. Then for the quasihomogeneous (with $\varepsilon$ taken into account) operators $\mathcal{P}\left(D_{t}, D_{x}, \varepsilon\right):=\mathcal{P}\left(y^{0}, D_{t}, D_{x}, \varepsilon\right), \mathcal{Q}\left(D_{t}, D_{x}, \varepsilon\right):=\mathcal{Q}\left(y^{0}, D_{t}, D_{x}, \varepsilon\right)$ corresponding to an arbitrary point $y^{0} \in \mathbb{R}^{n+1}$ we have

$$
|\gamma| \cdot\|u\|_{m-N, N-1,[\gamma]}^{2} \leq-C \operatorname{Im}\left(\mathcal{P}\left(D_{t}, D_{x}, \varepsilon\right) u, \mathcal{Q}\left(D_{t}, D_{x}, \varepsilon\right) u\right)_{[\gamma]}, \quad \gamma \leq 0 .
$$

Suppose the condition $(\tilde{\mathrm{B}})$ of Theorem 7.2 holds. Then for each $y^{0} \in \mathbb{R}^{n+1}$ the polynomials $\mathcal{P}(\tau, \xi)$ and $\mathcal{Q}(\tau, \xi)$ have no common real zeros, and according to Lemma [8.1. (8.8) holds. In other words, condition (B) of Proposition 7.1] holds. This proposition implies (A).

Proof of Lemma 8.1. Take $\theta>0$, replace $\gamma$ and $\varepsilon$ in inequality (7.13) with $\frac{\gamma}{\theta}$ and $\theta \varepsilon$ respectively, and apply this inequality to the function

$$
u_{\theta}(y)=u\left(\frac{y-y^{0}}{\theta}\right), \quad u \in \mathcal{D} .
$$

Replacing $y$ with $y^{0}+\theta y, y^{0}=\left(t^{0}, x^{0}\right)$, in the integrand, we obtain on the left-hand side of (7.13) the expression

$$
\theta^{n+1} e^{\frac{\gamma t^{0}}{\theta}} \operatorname{Im} \int e^{2 \gamma t} \mathcal{P}\left(y^{0}+\theta y, \frac{D}{\theta}, \theta \varepsilon\right) u(y) \overline{\mathcal{Q}\left(y^{0}+\theta y, \frac{D}{\theta}, \theta \varepsilon\right) u(y)} d y .
$$

We now note that for $N$ even,

$$
\begin{aligned}
& \mathcal{P}\left(y^{0}+\theta y, \frac{D}{\theta}, \theta \varepsilon\right)=\theta^{-m+N} \mathcal{P}\left(y^{0}+\theta y, D, \varepsilon\right), \\
& \mathcal{Q}\left(y^{0}+\theta y, \frac{D}{\theta}, \theta \varepsilon\right)=\theta^{-m+N-1} \mathcal{Q}\left(y^{0}+\theta y, D, \varepsilon\right),
\end{aligned}
$$

and for $N$ odd,

$$
\begin{aligned}
& \mathcal{P}\left(y^{0}+\theta y, \frac{D}{\theta}, \theta \varepsilon\right)=\theta^{-m+N-1} \mathcal{P}\left(y^{0}+\theta y, D, \varepsilon\right), \\
& \mathcal{Q}\left(y^{0}+\theta y, \frac{D}{\theta}, \theta \varepsilon\right)=\theta^{-m+N} \mathcal{Q}\left(y^{0}+\theta y, D, \varepsilon\right) .
\end{aligned}
$$

Therefore, the integral (8.9) takes the form

$$
\theta^{n-2 m+2 N} e^{\frac{\gamma t^{0}}{\theta}} \operatorname{Im} \int e^{2 \gamma t} \mathcal{P}\left(y^{0}+\theta y, D, \varepsilon\right) u(y) \overline{\mathcal{Q}\left(y^{0}+\theta y, D, \varepsilon\right) u(y)} d y .
$$


Similar arguments show that

$$
\begin{aligned}
& \frac{|\gamma|}{\theta}\left\|\delta_{m-N}^{+}(D) \delta_{N-1}^{+}(\theta \varepsilon D) u_{\theta}\right\|_{[\gamma / \theta]}^{2} \\
& \quad=\theta^{n-2 m+2 N} e^{\frac{\gamma t^{0}}{\theta}} \int e^{2 \gamma t}\left|\left(i D+\sqrt{\theta^{2}+|\xi|^{2}}\right)^{m-N} \delta_{N-1}^{+}(\varepsilon D) u(y)\right|^{2} d y .
\end{aligned}
$$

Dividing (8.10) and (8.11) by the common factor and passing to the limit as $\theta \rightarrow 0$, we obtain (8.8).

\subsection{The proof of inequality (7.13). Reduction to the results of [11]. Denote}

$$
H_{\mathcal{P} \mathcal{Q}}(y ; \gamma, \eta, \varepsilon):=-\operatorname{Im} \mathcal{P}(y ; \tau, \xi, \varepsilon) \overline{\mathcal{Q}(y ; \tau, \xi, \varepsilon)} .
$$

Here and everywhere below we set $\tau=\sigma+i \gamma, \eta=(\sigma, \xi)$. In condition (A) of Theorem 7.2 the symbol (8.12) satisfies the positivity condition

$$
H_{\mathcal{P} \mathcal{Q}}(y ; \gamma, \eta, \varepsilon)>0, \quad \gamma<0
$$

and also the following constant strength condition.

For all $y^{\prime}, y^{\prime \prime} \in \mathbb{R}^{n+1}$ there exists a constant $K=K\left(y^{\prime}, y^{\prime \prime}\right)$ such that for $\gamma \leq 0$ we have

$$
K^{-1} \leq \frac{H_{\mathcal{P} \mathcal{Q}}\left(y^{\prime} ; \gamma, \eta, \varepsilon\right)}{H_{\mathcal{P} \mathcal{Q}}\left(y^{\prime \prime} ; \gamma, \eta, \varepsilon\right)} \leq K
$$

Choose a point $y^{0} \in \mathbb{R}^{n+1}$ and denote by $\mathcal{P}(\tau, \xi, \varepsilon), \mathcal{Q}(\tau, \xi, \varepsilon), H_{\mathcal{P} \mathcal{Q}}(\tau, \xi, \varepsilon)$ the values of the symbols under consideration at this point. Due to the constant strength condition, the choice of the point $y^{0}$ is irrelevant. Inequality (7.13) can be rewritten as an estimate from above and from below of the middle form in (7.13) in terms of similar forms with constant coefficients frozen at some point, say $y^{0}$ :

$$
\begin{gathered}
-C^{-1} \operatorname{Im}(\mathcal{P}(D, \varepsilon) u, \mathcal{Q}(D, \varepsilon) u)_{s[\gamma]} \leq-\operatorname{Im}(\mathcal{P}(y ; D, \varepsilon) u, \mathcal{Q}(y ; D, \varepsilon) u)_{s[\gamma]} \\
\leq-C \operatorname{Im}(\mathcal{P}(D, \varepsilon) u, \mathcal{Q}(D, \varepsilon) u)_{s[\gamma]}, \quad|s| \leq|S|, \quad \gamma \leq \gamma_{0}(S) \leq 0 .
\end{gathered}
$$

The question on the existence of the estimate (8.15) in the special case

$$
\mathcal{Q}(y ; \tau, \xi, \varepsilon)=\frac{\partial \mathcal{P}(y ; \tau, \xi, \varepsilon)}{\partial \tau}
$$

is considered in details in Chapter VII of the book [11] (of course, there the small parameter $\varepsilon$ is not singled out). A trivial modification of the proof of Proposition 1 in [11 VII.2.1] allows one to reduce the proof of (8.15) to the verification of certain conditions on the symbol.

We will use the following standard notation:

$$
A_{(\alpha)}^{(\beta)}(y ; \tau, \xi)=\partial_{(\tau, \xi)}^{\beta} D_{y}^{\alpha} A(y ; \tau, \xi) .
$$

In the cases $\alpha=0$ or $\beta=0$ the corresponding index will be omitted.

Proposition 8.1. Conditions (8.13), (8.14), and

$$
\begin{gathered}
\left|H_{\mathcal{P} \mathcal{Q}}^{(\beta)}(y ; \gamma, \eta, \varepsilon)\right| \leq \lambda_{\beta}(\gamma) H_{\mathcal{P} \mathcal{Q}}(y ; \gamma, \eta, \varepsilon), \quad \beta>0, \\
\left|\overline{\mathcal{Q}^{(\beta)}(y ; \tau, \xi, \varepsilon)} \mathcal{P}(y ; \tau, \xi, \varepsilon)-\overline{\mathcal{P}^{(\beta)}(y ; \tau, \xi, \varepsilon)} \mathcal{Q}(y ; \tau, \xi, \varepsilon)\right| \\
\leq \lambda_{\beta}(\gamma) H_{\mathcal{P} \mathcal{Q}}(y ; \gamma, \eta, \varepsilon), \quad \beta>0, \\
\left|\overline{\mathcal{Q}^{(\beta)}(y ; \tau, \xi, \varepsilon)} \mathcal{P}_{(\alpha)}(y ; \tau, \xi, \varepsilon)-\overline{\mathcal{P}^{(\beta)}(y ; \tau, \xi, \varepsilon)} \mathcal{Q}_{(\alpha)}(y ; \tau, \xi, \varepsilon)\right| \\
\leq \lambda_{\alpha \beta}(\gamma) H_{\mathcal{P} \mathcal{Q}}(y ; \gamma, \eta, \varepsilon)(1+|\gamma|+|\eta|)(1+|\xi|)^{|\alpha|-1}, \quad \beta \geq 0, \quad \alpha>0,
\end{gathered}
$$


hold, where by $\lambda_{\beta}(\gamma), \lambda_{\alpha \beta}(\gamma)$ we denote constants that tend to zero as $\gamma \rightarrow-\infty$.

Then for each $S>0$ there is $\gamma_{0}=\gamma_{0}(S)$ such that (8.15) holds for $|s| \leq S$ and $\gamma \leq \gamma_{0}$.

Remark 8.1. 1) The constant $C$ in (8.15) depends on the constant $K$ in (8.14) and on the constants $\lambda_{\beta}(\gamma), \lambda_{\alpha \beta}(\gamma)$ in (8.16)-(8.18). If all these constants are independent of the small parameter $\varepsilon$, then the constant $C$ in (8.15) also does not depend on the small parameter, and so we obtain an estimate that is uniform with respect to $\varepsilon$.

2) In Proposition 1 in [11. VII.2.1] the estimate (8.18) was made unnecessarily strong in that the factor $(1+|\gamma|+|\eta|)(1+|\xi|)^{|\alpha|-1}$ was replaced with $(1+|\xi|)^{|\alpha|}$. In turn, this required the independence of $y$ of the coefficients of the leading power of $\tau$ in $\mathcal{P}$ and $\mathcal{Q}$.

Main steps in the proof of Proposition 8.1] The deduction of the estimate (8.15) from the assumptions of Proposition 8.1 is rather long. For completeness, we concentrate on some key moments of the proof.

Setting $w=e^{\gamma t} \delta_{s}^{+}(D) u$ and $D_{\gamma}=\left(D_{t}+i \gamma, D_{x}\right)$, we rewrite the middle term in (8.15) in the following form (omitting the parameter $\varepsilon$, which is unessential in this context):

$$
\begin{aligned}
-\operatorname{Im}\left(\delta_{s}^{+}\left(D_{\gamma}\right) \mathcal{P}\left(y ; D_{\gamma}\right) \delta_{-s}^{+}\left(D_{\gamma}\right) w, \delta_{s}^{+}\left(D_{\gamma}\right) \mathcal{O}\left(y ; D_{\gamma}\right) \delta_{-s}^{+}\left(D_{\gamma}\right) w\right)_{s[\gamma]} \\
=\left(\frac { i } { 2 } \delta _ { - s } ^ { + } ( D _ { - \gamma } ) \left[\mathcal{Q}^{*}\left(y ; D_{-\gamma}\right) \delta_{s}^{+}\left(D_{\gamma}\right) \delta^{+}\left(D_{-\gamma}\right) \mathcal{P}\left(y ; D_{\gamma}\right)\right.\right. \\
\left.\left.\quad-\mathcal{P}^{*}\left(y ; D_{-\gamma}\right) \delta_{s}^{+}\left(D_{\gamma}\right) \delta_{s}^{+}\left(D_{-\gamma}\right) \mathcal{Q}\left(y ; D_{\gamma}\right)\right] \delta_{-s}^{+}\left(D_{\gamma}\right) w, w\right) \\
:=\operatorname{Re}\left(H_{\mathcal{P} \mathcal{Q}}(y ; \gamma, D) w, w\right)+\left(G_{s \gamma} w, w\right) .
\end{aligned}
$$

The estimate 8.15 is a direct consequence of the following two inequalities:

$$
\begin{gathered}
C^{-1}\left(H_{\mathcal{P} \mathcal{Q}}(\gamma, D) w, w\right) \leq \operatorname{Re}\left(H_{\mathcal{P} \mathcal{Q}}(y ; \gamma, D) w, w\right) \leq C\left(H_{\mathcal{P} \mathcal{Q}}(\gamma, D) w, w\right), \\
\left(G_{s \gamma} w, w\right) \leq \lambda_{s}(\gamma)\left(H_{\mathcal{P} \mathcal{Q}}(\gamma, D) w, w\right) .
\end{gathered}
$$

The right inequality in (8.19) follows immediately from the constant strength condition. The same is true for the left inequality in the case where the coefficients are close to constant. Using a partition of unity, the general case is reduced to the case where the coefficients are close to constant. The resulting forms correspond to the commutators of the operator $H_{\mathcal{P} \mathcal{Q}}(\gamma, D)$ and the function that appear in the partition of unity, and can be estimated using conditions (8.16) (see [11, VII.2.6] for more details).

As for inequality (8.20), it is based on the fact that $G_{s \gamma}$ is a pseudodifferential operator with the symbol (see [11, VII.2.3 and VII.2.4])

$$
\sum_{|\alpha+\beta+\theta|} \frac{1}{\alpha ! \beta ! \theta !}\left(\overline{\mathcal{Q}^{(\beta)}} \mathcal{P}_{(\alpha)}-\overline{\mathcal{P}^{(\beta)}} \mathcal{Q}_{(\alpha)}\right)_{(\beta+\theta)}\left(\delta_{s}^{+} \delta_{s}^{-}\right)^{(\alpha)} \delta_{-s}^{+} \delta_{-s}^{-(\theta)}-\frac{1}{2} \sum_{\beta>0} \frac{1}{\beta !} H_{\mathcal{P} \mathcal{Q}(\beta)}^{(\beta)}
$$

Denote by $G_{s M}(y ; \gamma, \eta)$ the symbol obtained by replacing the generically infinite sum (8.21) with the finite sum of the terms such that $|\alpha+\beta+\theta| \leq M$. Conditions (8.16) $-(8.18)$ yield the inequality

$$
\left|G_{s M}(y ; \gamma, \eta)\right|<\lambda_{M}(\gamma) H_{\mathcal{P} \mathcal{Q}}(\gamma, \eta), \quad \lambda_{M}(\gamma) \rightarrow 0, \quad \gamma \rightarrow-\infty .
$$

The inequality for the symbols implies the inequality for the forms:

$$
\left|\left(G_{s M}(y ; \gamma, D) w, w\right)\right|<\lambda_{M}^{\prime}(\gamma)\left(H_{\mathcal{P} \mathcal{Q}}(\gamma, D) w, w\right) .
$$

Separately, one estimates the remainder resulting from replacing the infinite series with the finite sum (see [11, Lemma 3 in VII.2.4]). 
8.4. Verification of the assumptions of Proposition 8.1 for symbols satisfying the assumptions of Theorem 7.2, The next (rather rough) result significantly simplifies the required verification.

Lemma 8.2. Let the constant strength condition be satisfied, and suppose that for all $y^{\prime}, y^{\prime \prime} \in \mathbb{R}^{n+1}$ we have

$$
\begin{gathered}
\left|\mathcal{Q}^{\left(\beta^{\prime}\right)}\left(y^{\prime}, \gamma, \eta\right)\right| \cdot\left|\mathcal{P}^{\left(\beta^{\prime \prime}\right)}\left(y^{\prime \prime}, \gamma, \eta\right)\right|<\lambda_{\beta^{\prime} \beta^{\prime \prime}}(\gamma) H_{\mathcal{P} \mathcal{Q}}(\gamma, \eta), \quad\left|\beta^{\prime}+\beta^{\prime \prime}\right|>0, \\
\left|\mathcal{Q}\left(y^{\prime}, \gamma, \eta\right)\right| \cdot\left|\mathcal{P}\left(y^{\prime \prime}, \gamma, \eta\right)\right|<\lambda(\gamma) H_{\mathcal{P} \mathcal{Q}}(\gamma, \eta)(1+|\gamma|+|\eta|),
\end{gathered}
$$

where $\lambda_{\beta^{\prime} \beta^{\prime \prime}}(\gamma), \lambda(\gamma) \rightarrow 0, \gamma \rightarrow-\infty$.

Then all assumptions of Proposition 8.1 are satisfied.

Proof. First we note that

$$
H_{\mathcal{P Q}}^{(\beta)}=-\operatorname{Im} \sum_{\beta^{\prime}+\beta^{\prime \prime}=\beta} \frac{\left(\beta^{\prime}+\beta^{\prime \prime}\right) !}{\beta^{\prime} ! \beta^{\prime \prime} !} \mathcal{P}^{\left(\beta^{\prime}\right)} \overline{\mathcal{Q}^{\left(\beta^{\prime \prime}\right)}},
$$

and the estimate (8.4) follows from (8.22).

In verifying (8.17) and (8.18) we use the fact that, due to the finite dimensionality of the space of polynomials and the stabilization at infinity condition, we can find a finite set of points $y^{1}, \ldots, y^{J} \in \mathbb{R}^{n+1}$, such that

$$
\begin{aligned}
& \mathcal{P}(y ; \tau, \xi, \varepsilon)=\sum_{j=1}^{J} p_{j}(y) \mathcal{P}\left(y^{j} ; \tau, \xi, \varepsilon\right), \\
& \mathcal{Q}(y ; \tau, \xi, \varepsilon)=\sum_{j=1}^{J} q_{j}(y) \mathcal{Q}\left(y^{j} ; \tau, \xi, \varepsilon\right)
\end{aligned}
$$

with smooth coefficients $p_{j}(y)$ and $q_{j}(y)$. Now we can rewrite the left-hand side of (8.17) as follows:

$$
\left|\sum_{j, k=1}^{J} p_{j}(y) q_{k}(y)\left(\overline{\mathcal{Q}^{(\beta)}\left(y^{k}\right)} \mathcal{P}\left(y^{j}\right)-\overline{\mathcal{P}^{(\beta)}\left(y^{j}\right)} \mathcal{Q}\left(y^{k}\right)\right)\right| .
$$

Using (8.22), we obtain (8.17).

To verify (8.18), we note that the expression on the left-hand side is the value at $y^{\prime}=y^{\prime \prime}=y$ of the expression

$$
\sum q_{j}\left(y^{\prime}\right) D_{y^{\prime \prime}}^{\alpha} p_{k}\left(y^{\prime \prime}\right) \overline{\mathcal{Q}^{(\beta)}\left(y^{j}\right)} \mathcal{P}\left(y^{k}\right)-\sum p_{j}\left(y^{\prime}\right) D_{y^{\prime \prime}}^{\alpha} q_{k}\left(y^{\prime \prime}\right) \overline{\mathcal{Q}^{(\beta)}\left(y^{j}\right)} \mathcal{P}\left(y^{k}\right) .
$$

For $\beta>0$ this expression can be estimated using (8.22). For $\beta=0$ one should use (8.23).

Now we verify that conditions (8.22) and (8.23) hold for symbols satisfying, for each $y$, conditions $(\mathrm{I}),\left(\mathrm{II}_{0}\right),\left(\mathrm{III}_{0}\right)$ and $(\mathrm{IV})$.

According to definitions in 6.1 , both for even and for odd $N$ we have

$$
\mathcal{Q}^{\left(\beta^{\prime}\right)} \mathcal{P}^{\left(\beta^{\prime \prime}\right)}=\sum(-1)^{j+k} \gamma_{2 j+1} \gamma_{2 j} \varepsilon^{2 N-2 j-2 k-2} P_{2 j+1}^{\left(\beta^{\prime}\right)} P_{2 k}^{\left(\beta^{\prime \prime}\right)}
$$

Therefore,

$$
\left|\mathcal{Q}^{\left(\beta^{\prime}\right)} \mathcal{P}^{\left(\beta^{\prime \prime}\right)}\right| \leq \text { const } \sum \varepsilon^{2 N-2 j-2 k-2}(|\tau|+|\xi|)^{2 m-2 j-2 k-1-\left|\beta^{\prime}+\beta^{\prime \prime}\right|} .
$$

If $\left|\beta^{\prime}+\beta^{\prime \prime}\right| \geq 1$, then the right-hand side of this formula does not exceed

$$
C(|\tau|+|\xi|)^{2 m-2 N}(1+\varepsilon(|\tau|+|\xi|))^{2 N-2} .
$$


This expression can be estimated by the left-hand side of (6.6) with the constant $(C|\gamma|)^{-1}$, and by Theorem 6.1 it is estimated by $H_{\mathcal{P} \mathcal{Q}}$ with another constant $\frac{C^{\prime}}{|\gamma|}$. For $\beta^{\prime}=\beta^{\prime \prime}=0$ we obtain

$$
(|\tau|+|\xi|)^{-1}|\mathcal{Q P}| \leq \operatorname{const}(|\tau|+|\xi|)^{m-2 N}(1+\varepsilon(|\tau|+|\xi|))^{2 N-2} .
$$

The proof of Theorem 7.2 is completed.

\section{Pencils Related to the system of moments for the Kinetic FOKKER-PLANCK EQUATION}

9.1. The Fokker-Planck equation and Hermite functions. The classical Boltzmann equation for the particle distribution function $f(t, x, c), x \in \mathbb{R}^{n}, c \in \mathbb{R}^{n}$ (velocities), $n=1,2,3$, has the form

$$
\left(\partial_{t}+c_{k} \partial_{x_{k}}\right) f(t, x, c)=S(f),
$$

where $S(f)$ is the so-called collision integral. As usual, we assume summation over repeating indices. The collision operator takes the simplest form in the case of the Fokker-Planck equation describing the distribution of the number of Brownian particles:

$$
S(f)=\partial_{c_{k}}\left(c_{k}+\partial_{c_{k}}\right) f .
$$

The corresponding equation is called the kinetic Fokker-Planck equation,

$$
\left(\partial_{t}+c_{k} \partial_{x_{k}}\right) f(t, x, c)=\partial_{c_{k}}\left(c_{k}+\partial_{c_{k}}\right) f(t, x, c) .
$$

The equilibrium distribution $f_{E}(c)$ (the Maxwell distribution) is the unique (up to a multiplicative constant) annihilator of the collision operator:

$$
S\left(f_{E}\right)=0 \rightarrow f_{E}(c)=(2 \pi)^{-n / 2} e^{-\frac{|c|^{2}}{2}},
$$

which does not depend on spatial variables and on time.

The Grad method for solving the kinetic equation (9.1) consists in looking for the solution as the result of application of an infinite order differential operator to the equilibrium distribution:

$$
f(t, x, c)=\sum_{|\alpha| \geq 0} \frac{1}{\alpha !} m^{\alpha}(x, t)\left(-\partial_{c}\right)^{\alpha} f_{E}(c),
$$

where the coefficients $m^{\alpha}(x, t)$ depend of the spatial variable and on time and have to be determined.

The functions

$$
\psi^{\alpha}(c)=\left(-\partial_{c}\right)^{\alpha} f_{E}(c)
$$

are called the (multidimensional) Hermite functions. Therefore, (9.2) is nothing else but a representation of the unknown function $f(t, x, c)$ as a series in Hermite functions in variables $c$ :

$$
f(t, x, c)=\sum_{|\alpha| \geq 0} \frac{1}{\alpha !} m^{\alpha}(x, t) \psi^{\alpha}(c) .
$$

Substituting (9.3) in the Fokker-Planck equation (9.1) we obtain

$$
\sum_{|\alpha| \geq 0} \frac{1}{\alpha !}\left(\partial_{t} m^{\alpha}(x, t)+c_{k} \partial_{x_{k}} m^{\alpha}(x, t)\right) \psi^{\alpha}=\sum_{|\alpha| \geq 0} \frac{1}{\alpha !} m^{\alpha}(x, t) \partial_{c_{k}}\left(c_{k}+\partial_{c_{k}}\right) \psi^{\alpha}(c) .
$$

Since the Hermite functions form a complete system, the equality of series in (9.4) implies relations for the coefficients. To deduce these relations, we will need some simple facts about the Hermite functions. 
9.2. Remarks on the multidimensional Hermite functions. The functions $\psi^{\alpha}$ form a complete orthonormal system in the Hilbert function space $L_{f_{E}}^{2}\left(\mathbb{R}^{n}\right)$ with weight $\frac{1}{f_{E}}$ :

$$
\left(\psi^{\alpha}, \psi^{\beta}\right)=\int_{\mathbb{R}^{n}} \psi^{\alpha}(c) \psi^{\beta}(c) \frac{d c}{f_{E}(c)}=\alpha ! \delta_{\alpha \beta} .
$$

We will need certain recurrence relations for the Hermite functions. Denoting by $e_{j}, j=1, \ldots, n$, the unit vectors in $\mathbb{R}^{n}$, we have

$$
\begin{aligned}
\psi^{\alpha+e_{j}}(c)+\partial_{c_{j}} \psi^{\alpha}(c) & =0, \\
\psi^{\alpha+e_{j}}(c)+\alpha_{j} \psi^{\alpha-e_{j}}(c)-c_{j} \psi^{\alpha}(c) & =0, \\
\partial_{c_{j}} \psi^{\alpha}(c)+c_{j} \psi^{\alpha}(c)-\alpha_{j} \psi^{\alpha-e_{j}}(c) & =0, \\
\partial_{c_{j}}^{2} \psi^{\alpha}(c)+c_{j} \partial_{c_{j}} \psi^{\alpha}(c)+\left(1+\alpha_{j}\right) \psi^{\alpha}(c) & =0 .
\end{aligned}
$$

Formula (9.5) immediately follows from the definition of functions $\psi^{\alpha}$; 9.7) follows from (9.6) in view of (9.5), and in verifying (9.6) and (9.8) it is sufficient to consider functions $\psi^{k}$ of one variable $c$ :

$$
\psi^{k+1}(c)=(-\partial)^{k}\left(-\partial e^{-\frac{c^{2}}{2}}\right)=(-\partial)^{k}\left(c e^{-\frac{c^{2}}{2}}\right)=c \psi^{k}-k \psi^{k-1}
$$

(the last equality follows from the Leibniz formula). Similarly,

$$
\begin{aligned}
\partial^{2} \psi^{k}(c) & =(-1)^{k+1} \partial^{k+1}\left(c e^{-\frac{c^{2}}{2}}\right) \\
& =(-1)^{k+1}\left(c \partial^{k+1} e^{-\frac{c^{2}}{2}}+(k+1) \partial^{k} e^{-\frac{c^{2}}{2}}\right)=c \psi^{k+1}-(k+1) \psi^{k} .
\end{aligned}
$$

9.3. The infinite system for the coefficient $m^{\alpha}$. By the completeness of the system of Hermite functions $\psi^{\alpha}$, formula (9.4) is equivalent to an infinite system of equalities obtained by taking inner products of both sides of (9.4) with the function $\psi^{\beta}$ for all possible $\beta$. For a fixed $\beta$ the left-hand side of (9.4) becomes

$$
\partial_{t} m^{\beta}(x, t)+\sum_{\alpha} \frac{1}{\alpha !} \partial_{x_{k}} m^{\alpha}(x, t)\left(c_{k} \psi^{\alpha}, \psi^{\beta}\right) .
$$

By (9.6),

$$
\begin{aligned}
\sum_{\alpha} \frac{1}{\alpha !}\left(c_{k} \psi^{\alpha}, \psi^{\beta}\right) \partial_{x_{k}} m^{\alpha}(x, t) & =\sum_{\alpha} \frac{1}{\alpha !}\left(\psi^{\alpha+e_{k}}+\alpha_{k} \psi^{\alpha-e_{k}}, \psi^{\beta}\right) \partial_{x_{k}} m^{\alpha}(x, t) \\
& =\beta_{k} \partial_{x_{k}} m^{\beta-e_{k}}(x, t)+\partial_{x_{k}} m^{\beta+e_{k}}(x, t) .
\end{aligned}
$$

Let us look at the right-hand side. Note that according to (9.8),

$$
\partial_{c_{k}}\left(c_{k}+\partial_{c_{k}}\right) \psi^{\alpha}=\partial_{c_{k}}^{2} \psi^{\alpha}+c_{k} \partial_{c_{k}} \psi^{\alpha}+\psi^{\alpha}=-\alpha_{k} \psi^{\alpha} .
$$

Therefore,

$$
\sum_{\alpha} \frac{1}{\alpha !} m^{\alpha}(x, t)\left(\partial_{c_{k}}\left(c_{k}+\partial_{c_{k}}\right) \psi^{\alpha}, \psi^{\beta}\right)=-\sum_{k} \beta_{k} m^{\beta}(x, t)=-|\beta| m^{\beta}(x, t) .
$$

Hence, the Fokker-Planck equation can be rewritten as an infinite system of equations

$$
\partial_{t} m^{\beta}(x, t)+\beta_{k} \partial_{x_{k}} m^{\beta-e_{k}}(x, t)+\partial_{x_{k}} m^{\beta+e_{k}}(x, t)+|\beta| m^{\beta}(x, t)=0 .
$$

In the special case $n=1$ the system (9.10) takes a particularly simple form:

$$
\partial_{t} m^{l}(x, t)+l \partial_{x} m^{l-1}(x, t)+\partial_{x} m^{l+1}(x, t)+l m^{l}(x, t)=0, \quad l=0,1, \ldots .
$$


9.4. The Galerkin method for the system (9.10). We look for an approximate solution of the system (9.10) in the form of a finite sum

$$
f^{N}(t, x, c)=\sum_{0 \leq|\alpha| \leq N} \frac{1}{\alpha !} m^{\alpha}(x, t)\left(-\partial_{c}\right)^{\alpha} f_{E}(c) .
$$

We take inner products corresponding to $|\beta| \leq N$. In this case the equations corresponding to $|\beta|<N$ do not change, and the equations corresponding to $|\beta|=N$ take the form

$$
\partial_{t} m^{\beta}(x, t)+\beta_{k} \partial_{x_{k}} m^{\beta-e_{k}}(x, t)+|\beta| m^{\beta}(x, t)=0 .
$$

Introducing the vector-valued function $m(x, t)=\left\{m^{\beta}(x, t), 0 \leq|\beta| \leq N\right\}$, we obtain the following first order system:

$$
\partial_{t} m(x, t)+\sum_{j} A_{j} \partial_{x_{j}} m(x, t)+B m(x, t)=0,
$$

where

$$
A_{j}=\left(a_{j}^{\alpha, \beta}\right), \quad j=1, \ldots, n, \quad B=\left(b^{\alpha, \beta}\right),
$$

$B$ is a diagonal matrix (i.e., $b^{\alpha, \beta}=|\alpha| \delta_{\alpha, \beta}$ ) and the only nonzero elements of the matrix $A$ are

$$
a_{j}^{\alpha-e_{j}, \alpha}=\alpha_{j}, \quad a_{j}^{\alpha+e_{j}, \alpha}=1 .
$$

Replacing $\partial_{t}$ with $D_{t}=i^{-1} \partial_{t}$ and $\partial_{x_{j}}$ with $D_{j}=i^{-1} \partial_{x_{j}}$, we rewrite the system (9.12) in the form

$$
D_{t} m(x, t)+\sum_{j} A_{j} D_{j} m(x, t)-i B m(x, t)=0 .
$$

The algebraic equation

$$
P(\tau, \xi):=\operatorname{det}\left(\tau I+\sum A_{j} \xi_{j}-i B\right)=0
$$

is called the dispersion relation for the equation (9.12). Our problem is to study the polynomial on the left-hand side of (9.14). Setting $m=N+1$, we rewrite our polynomial in the form

$$
P(\tau, \xi):=\sum_{j=0}^{m}(-i)^{j} P_{j}(\tau, \xi),
$$

where $P_{j}(\tau, \xi)$ are real homogeneous polynomials of degree $m-j, j=0, \ldots, m$. We start with a few simple comments.

1) $P_{m}(\tau, \xi) \equiv 0$. Indeed, $P_{m}(\tau, \xi)$ is a homogeneous polynomial of degree 0 independent of the variables $(\tau, \xi)$. But then

$$
P_{m}(\tau, \xi)=P_{m}(0,0)=\operatorname{det}(-i B)=0 .
$$

Because of this, we rewrite the polynomials (9.15) in the form

$$
P(\tau, \xi):=\sum_{j=0}^{N}(-i)^{j} P_{j}(\tau, \xi)
$$

2) Let $\gamma_{j}=P_{j}(1,0)$. Then the constants $\gamma_{j}$ are positive and satisfy the Routh-Hurwitz conditions. Indeed, by definition,

$$
P(\tau, 0)=\sum_{j=0}^{N}(-i)^{j} \gamma_{j} \tau^{m-j}=\operatorname{det}(\tau I-i B)=\tau \prod_{j=1}^{N}(\tau-i j) .
$$


The roots of the polynomial $\prod_{j=1}^{N}(\tau-i j)$ belong to the upper complex half-plane, and so its coefficients satisfy the Routh-Hurwitz conditions.

3) We have

$$
\gamma_{N} P_{N}(\tau, \xi)=N ! \tau
$$

Indeed, the polynomial $P(\tau, \xi)$ can be viewed as the value at $\lambda=1$ of the homogeneous polynomial

$$
P(\tau, \xi, \lambda):=\sum_{j=0}^{N}(-i)^{j} \gamma_{j} \lambda^{j} P_{j}(\tau, \xi)=\operatorname{det}(\tau I-i \lambda B) .
$$

Comparing the coefficients of $(-i \lambda)^{N}$, we arrive at (9.18).

4) The polynomials $P_{N-1}$ and $P_{N}$ form a strictly hyperbolic pair.

The verification of this statement is based on explicit computation of the expression $\gamma_{N-1} P_{N-1}$, which is the coefficient at $(-i \lambda)^{N-1}$ in the expansion of the polynomial (9.19) in powers of $-i \lambda$. For simplicity, we only consider the one-dimensional case.

Denote by $A_{i j}=A_{i j}(\tau, \xi, \lambda)$ the elements of the matrix $\tau I-i B-\xi A$. The polynomial (9.19) is the sum

$$
\sum \pm A_{1 i_{1}} \cdots A_{N+1, i_{N+1}}=A_{11} \cdots A_{N+1, N+1}+^{\prime} \sum \pm A_{1 i_{1}} \cdots A_{N+1, i_{N+1}}
$$

where the sum on the left-hand side is taken over all permutations of the numbers $1,2, \ldots, N+1$, and the sum on the right-hand side is over all permutations except the identity.

The first term on the right-hand side of (9.20) is equal to

$$
\tau \prod_{j=1}^{N}(\tau-i j \lambda)=N ! \tau(-i \lambda)^{N}+N ! \sum_{k=2}^{N+1} \frac{1}{k-1} \tau^{2}(-i \lambda)^{N-1}+O\left(\lambda^{N-2}\right) .
$$

The contribution to the coefficient of $(-i \lambda)^{N-1}$ on the right-hand side comes only from the terms that contain all diagonal elements $A_{22}, \ldots, A_{N+1, N+1}$ but one. Therefore, in computing the coefficient of $(-\lambda)^{N-1}$ we can replace the sum on the right-hand side in (9.20) with

$$
-\sum_{k} A_{1 k} A_{k 1} \prod_{2 \leq j \leq N+1, j \neq k} A_{j j}
$$

which is equal to

$$
-\sum_{k} a_{1 k} a_{k 1} \prod_{2 \leq j \leq N+1, j \neq k}(j-1)(-i \lambda)^{N-1}+O\left(\lambda^{N-2}\right),
$$

where $a_{k l}$ are the elements of the matrix $A$. Since the matrix $A$ is two-diagonal, the product $a_{1 k} a_{k 1}$ is different from zero only for $k=2$, in which case it is equal to $|\xi|^{2}$. Finally, we have

$$
\gamma_{N-1} P_{N-1}=N ! \sum_{k=2}^{N+1} \frac{1}{k-1} \tau^{2}-N !|\xi|^{2}
$$

For $\xi \neq 0$ the polynomial on the right-hand side has one positive and one negative root. The root $\tau=0$ separates these roots. 


\subsection{Examples.}

Example 9.1. In the case $n=1$ and $N=4$ that matrices $A$ and $B$ have the form

$$
A=\left(\begin{array}{llll}
0 & 1 & 0 & 0 \\
1 & 0 & 1 & 0 \\
0 & 2 & 0 & 1 \\
0 & 0 & 3 & 0
\end{array}\right), \quad B=\left(\begin{array}{cccc}
0 & 0 & 0 & 0 \\
0 & 1 & 0 & 0 \\
0 & 0 & 2 & 0 \\
0 & 0 & 0 & 3
\end{array}\right)
$$

The left-hand side of the dispersion equation

$$
\operatorname{det}(\tau I+\xi A-i B)=0
$$

equals

$$
P_{0}(\tau, \xi)-i \gamma_{1} P_{1}(\tau, \xi)-\gamma_{2} P_{2}(\tau, \xi)+i P_{3}(\tau, \xi)=0,
$$

where $\gamma_{1}=6, \gamma_{2}=10, \gamma_{3}=3$, and

$$
P_{0}=\tau^{4}-6 \tau^{2} \xi^{2}+3 \xi^{4}, \quad P_{1}=\tau\left(\tau^{2}-\frac{5}{6} \xi^{2}\right), \quad P_{2}=\tau^{2}-\frac{3}{5} \xi^{2}, \quad P_{3}=\tau .
$$

One can easily verify that all polynomials are strictly hyperbolic and the sets of roots of two neighboring polynomials strictly separate one another. For the constants $\gamma_{j}$ the Routh-Hurwitz condition holds. Elementary computations show that condition (5.7) also holds. Indeed,

$$
\left[P_{0}, P_{3}\right]=3 \tau^{4}-6 \tau^{2} \xi^{2}-3 \xi^{4}, \quad\left[P_{1}, P_{2}\right]=\frac{1}{6} \tau^{4}+\frac{11}{30} \tau^{2} \xi^{2} .
$$

Hence

$$
\gamma_{1} \gamma_{2}\left[P_{1}, P_{2}\right]-\gamma_{3}\left[P_{0}, P_{3}\right]=\tau^{4}+40 \tau^{2} \xi^{2}+27 \xi^{4}>0 .
$$

Therefore, condition (5.7) holds, so that all conditions of Theorem 4.2 hold. Applying this theorem, we prove the stability of the polynomial (9.22).

Example 9.2. Consider the representation of the Fokker-Planck equation in the basis of Hermite functions up to (and including) the first order. In dimension $n=3$ we obtain a system of four equations,

$$
\left\{\begin{array}{l}
\partial_{t} m^{000}+\partial_{x_{1}} m^{100}+\partial_{x_{2}} m^{010}+\partial_{x_{3}} m^{001}=0 \\
\partial_{t} m^{100}+\partial_{x_{1}} m^{000}+m^{100}=0 \\
\partial_{t} m^{010}+\partial_{x_{2}} m^{000}+m^{010}=0 \\
\partial_{t} m^{001}+\partial_{x_{3}} m^{000}+m^{001}=0
\end{array}\right.
$$

for the first four moments: the zero order moment $m^{000}$ and the three first order moments $m^{100}, m^{010}, m^{001}$.

The dispersion relation for this system determines a nonstrictly hyperbolic pencil of order $(4,3)$ :

$$
\tau^{2}\left(\tau^{2}-|\xi|^{2}\right)-i 3 \tau\left(\tau^{2}-\frac{2}{3}|\xi|^{2}\right)-3\left(\tau^{2}-\frac{1}{3}|\xi|^{2}\right)+i \tau
$$

where the coefficients $\gamma_{1}=\gamma_{2}=3, \gamma_{3}=1$ satisfy the Routh-Hurwitz condition, the polynomials $P_{0}$ and $P_{1}$ form a nonstrictly hyperbolic pair, and $P_{1}, P_{2}$ and $P_{2}, P_{3}$ are a strictly hyperbolic pair. Now we verify that the condition (5.7) holds, i.e., the polynomial (9.23) is stable. We have

$$
\gamma_{1} \gamma_{2}\left[P_{1}, P_{2}\right]=9\left(\tau^{4}-\frac{2}{3} \tau^{2}|\xi|^{2}+\frac{2}{9}|\xi|^{4}\right), \quad \gamma_{3}\left[P_{0}, P_{3}\right]=3 \tau^{4}-\tau^{2}|\xi|^{2} .
$$


The difference of these polynomials equals

$$
9 \tau^{4}-5 \tau^{2}|\xi|^{2}+2|\xi|^{4}=\left(\tau^{2}-\frac{5}{6}|\xi|^{2}\right)^{2}+\frac{47}{36}|\xi|^{4}>0 .
$$

Example 9.3. In the two-dimensional case $n=2$ the Grad method applied to the first six moments results in a $6 \times 6$ system with constant coefficients. The dispersion equation for this system takes the form

$$
\operatorname{det}\left(\begin{array}{cccccc}
\tau & \xi_{1} & \xi_{2} & 0 & 0 & 0 \\
\xi_{1} & \tau-i & 0 & 2 \xi_{1} & \xi_{2} & 0 \\
\xi_{2} & 0 & \tau-i & 0 & \xi_{1} & 2 \xi_{2} \\
0 & \xi_{1} & 0 & \tau-2 i & 0 & 0 \\
0 & \xi_{2} & \xi_{1} & 0 & \tau-2 i & 0 \\
0 & 0 & \xi_{2} & 0 & 0 & \tau-2 i
\end{array}\right)=0 .
$$

The left-hand side of this equation is the pencil

$$
P_{0}-\gamma_{2} P_{2}+\gamma_{4} P_{4}-i\left(\gamma_{1} P_{1}-\gamma_{3} P_{3}+\gamma_{5} P_{5}\right)
$$

where

$$
\begin{aligned}
& P_{0}\left(\tau, \xi_{1}, \xi_{2}\right)=\tau^{2}\left(\tau^{4}-4 \tau^{2}\left(\xi_{1}^{2}+\xi_{2}^{2}\right)+3\left(\xi^{1}+\xi_{2}^{2}\right)^{2}\right), \\
& P_{1}\left(\tau, \xi_{1}, \xi_{2}\right)=\tau\left(\tau^{4}-\frac{11}{4} \tau^{2}\left(\xi_{1}^{2}+\xi_{2}^{2}\right)+\left(\xi_{1}^{2}+\xi_{2}^{2}\right)^{2}\right), \\
& P_{2}\left(\tau, \xi_{1}, \xi_{2}\right)=\tau^{4}-\frac{42}{25} \tau^{2}\left(\xi_{1}^{2}+\xi_{2}^{2}\right)+\frac{4}{25}\left(\xi_{1}^{2}+\xi_{2}^{2}\right)^{2}, \\
& P_{3}\left(\tau, \xi_{1}, \xi_{2}\right)=\tau^{3}-\frac{16}{19} \tau\left(\xi_{1}^{2}+\xi_{2}^{2}\right) \\
& P_{4}\left(\tau, \xi_{1}, \xi_{2}\right)=\tau^{2}-\frac{2}{7}\left(\xi_{1}^{2}+\xi_{2}^{2}\right), \\
& P_{5}\left(\tau, \xi_{1}, \xi_{2}\right)=\tau .
\end{aligned}
$$

As for the coefficients $\gamma_{1}, \ldots, \gamma_{5}$, we have

$$
\gamma_{1}=8, \quad \gamma_{2}=25, \quad \gamma_{3}=38, \quad \gamma_{4}=28, \quad \gamma_{5}=8 .
$$

One can easily see that the polynomial $P_{0}$ is nonstrictly hyperbolic and the remaining polynomials $P_{j}, j=1, \ldots, 5$, are strictly hyperbolic. The polynomials $P_{0}, P_{1}$ form a nonstrictly hyperbolic pair, whereas the pairs $P_{1}, P_{2} ; P_{2}, P_{3} ; P_{3}, P_{4}$; and $P_{4}, P_{5}$ are strictly hyperbolic. The question on the stability of the polynomial (9.24) remains open.

9.6. Concluding remarks. As we have seen, direct computation of the polynomial pencils of dispersion equations for moment approximations of the Fokker-Planck equation shows that they satisfy rather strict conditions:

I. All polynomials in the pencil are strictly or nonstrictly hyperbolic. As has been shown earlier, they are resolved with respect to the leading power of $\tau$, and the leading coefficients satisfy the Routh-Hurwitz conditions.

II. The sets of roots of two neighboring polynomials separate one another, usually nonstrictly, i.e., two neighboring polynomials form a nonstrictly hyperbolic pair.

III. All elementary divisors of the characteristic matrix $I \tau+\sum_{j=1}^{n} A^{j} \xi_{j}$ for the moments are of degree one, and the number of equal elementary divisors does not depend on $\xi \in \mathbb{R}^{n}$.

It would be interesting to give a strict proof of these statements. Using III, one can show that $P_{0}, P_{1}$ form a nonstrictly hyperbolic pair. 


\section{REFERENCES}

[1] H. Grad, On the kinetic theory of rarefied gases. Comm. Pure Appl. Math. 2 (1949), no. 4, 331-406. MR0033674 (11:473a)

[2] E. V. Radkevich, Well-posedness of mathematical models in continuum mechanics and thermodynamics. Contemporary Mathematics. Fundamental Directions. 2003. Vol. 3. pp. 5-32.

[3] M. M. Postnikov, Stable polynomials, URSS, Moscow, 2004. (Russian)

[4] L. R. Volevich and M. G. Dzhavadov, Uniform estimates for solutions of hyperbolic equations with a small parameter multiplying the highest derivatives, Differentsial'nye Uravneniya 19 (1983), no. 12, 2082-2090; English transl. in Differential Equations 19 (1983). MR0729566 (85j:35020)

[5] L. R. Volevich and E. V. Radkevich, Uniform estimates for solutions of the Cauchy problem for hyperbolic equations with a small parameter at the highest derivative, Differentsial'nye Uravneniya 39 (2003), no. 4, 486-499; English transl. in Differential Equations 39 (2003).

[6] Ch. Hermite, Oeuvres I. Paris, 1905, pp. 397-414.

[7] L. Hörmander, Linear partial differential operators, Academic Press, New York; Springer-Verlag, Berlin-Göttingen-Heidelberg, 1963. MR0161012 (28:4221)

[8] M. I. Vishik and L. A. Lyusternik, Regular degeneration and boundary layer for linear differential equations with small parameter, Uspehi Mat. Nauk 12 (1957) no. 5(77), 3-122; English transl., Amer. Math. Soc. Transl. Ser. 2, vol. 20, Amer. Math. Soc., Providence, RI, 1962, pp. 229-364. MR0096041 (20:2539)

[9] L. R. Volevich and S. G. Gindikin, Mixed problem for partial differential equations with quasihomogeneous principal part, Amer. Math. Soc., Providence, RI, 1996. MR1357662 (96j:35145)

[10] L. R. Volevich, The Vishik-Lyusternik method in general elliptic boundary value problems with small parameter, Keldysh Inst. Appl. Math. RAS, 2002, Preprint no. 26.

[11] L. R. Volevich and S. G. Gindikin, The method of Newton's polyhedron in the theory of partial differential equations, Kluwer, Dordrecht, 1992. MR.1256484 (95a:35001)

Keldysh Institute of Applied Mathematics, Russian Academy of Sciences, Moscow 125047, Russia

E-mail address: volevich@spp.keldysh.ru

Moscow State University, Mechanics and Mathematics Department, Moscow 119899, Russia

E-mail address: evrad@land.ru 